\title{
Dermal adipose tissue has high plasticity and undergoes reversible dedifferentiation in mice
}

\author{
Zhuzhen Zhang, ${ }^{1}$ Mengle Shao, ${ }^{1}$ Chelsea Hepler, ${ }^{1}$ Zhenzhen Zi, ${ }^{2}$ Shangang Zhao, ${ }^{1}$ Yu A. An, ${ }^{1}$ Yi Zhu, ${ }^{1}$ Alexandra L. Ghaben, ${ }^{1}$ \\ May-yun Wang, ${ }^{1} \mathrm{Na} \mathrm{Li,}{ }^{1}$ Toshiharu Onodera, ${ }^{1}$ Nolwenn Joffin, ${ }^{1}$ Clair Crewe, ${ }^{1}$ Qingzhang Zhu, ${ }^{1}$ Lavanya Vishvanath, \\ Ashwani Kumar, ${ }^{3}$ Chao Xing, ${ }^{3}$ Qiong A. Wang, ${ }^{4}$ Laurent Gautron, ${ }^{5}$ Yingfeng Deng, ${ }^{1}$ Ruth Gordillo, ${ }^{1}$ Ilja Kruglikov, ${ }^{6}$ \\ Christine M. Kusminski, ${ }^{1}$ Rana K. Gupta, ${ }^{1}$ and Philipp E. Scherer ${ }^{1}$ \\ ${ }^{1}$ Touchstone Diabetes Center, ${ }^{2}$ Department of Biochemistry, and ${ }^{3}$ McDermott Center for Human Growth and Development, University of Texas Southwestern Medical Center, Dallas, Texas, USA \\ ${ }^{4}$ Department of Molecular and Cellular Endocrinology, Diabetes and Metabolism Research Institute, City of Hope/Beckman Research Institute, Duarte, California, USA. ${ }^{5}$ Division of Hypothalamic Research, \\ University of Texas Southwestern Medical Center, Dallas, Texas, USA. ${ }^{6}$ Scientific Department, Wellcomet GmbH, Karlsruhe, Germany.
}

\begin{abstract}
Dermal adipose tissue (also known as dermal white adipose tissue and herein referred to as dWAT) has been the focus of much discussion in recent years. However, dWAT remains poorly characterized. The fate of the mature dermal adipocytes and the origin of the rapidly reappearing dermal adipocytes at different stages remain unclear. Here, we isolated dermal adipocytes and characterized dermal fat at the cellular and molecular level. Together with dWAT's dynamic responses to external stimuli, we established that dermal adipocytes are a distinct class of white adipocytes with high plasticity. By combining pulse-chase lineage tracing and single-cell RNA sequencing, we observed that mature dermal adipocytes undergo dedifferentiation and redifferentiation under physiological and pathophysiological conditions. Upon various challenges, the dedifferentiated cells proliferate and redifferentiate into adipocytes. In addition, manipulation of dWAT highlighted an important role for mature dermal adipocytes for hair cycling and wound healing. Altogether, these observations unravel a surprising plasticity of dermal adipocytes and provide an explanation for the dynamic changes in dWAT mass that occur under physiological and pathophysiological conditions, and highlight the important contributions of dWAT toward maintaining skin homeostasis.
\end{abstract}

\section{Introduction}

White adipose tissue (WAT) evolved as the principal site for energy storage in vertebrate animals. White adipocytes, or "fat cells," are characterized by the presence of a single large lipid droplet ("unilocular" adipocytes). Their classical function is to serve as a storage compartment for excess energy; however, we now recognize that adipocytes are dynamic endocrine organs that secrete various hormones and cytokines ("adipokines") to control nutrient homeostasis, energy balance, and immune cell regulation (1). White adipocytes arise through the process of adipocyte differentiation, or "adipogenesis." In rodents and humans, most adipocytes arise pre- or perinatally, with fat cell numbers largely established by the adolescent period. In adulthood, there is relatively little fat cell turnover in the major, well-characterized, subcutaneous and intra-abdominal WAT depots. In response to certain challenges (e.g., caloric excess, cold exposure, injury), de novo fat cell differentiation can occur, through the recruitment of resident adipocyte precursors ("preadipocytes"). However, physiological changes in subcutaneous or intra-abdominal WAT mass are largely driven by alterations in lipid content (driven through lipogenesis and lipolysis) $(2,3)$.

Authorship note: Z. Zhang and M. Shao are co-first authors. Conflict of interest: The authors have declared that no conflict of interest exists. Copyright: @ 2019, American Society for Clinical Investigation. Submitted: May 13, 2019; Accepted: August 29, 2019; Published: October 28, 2019 Reference information: / Clin Invest. 2019;129(12):5327-5342. https://doi.org/10.1172/JCl130239.
Dermal adipose tissue (also known as dermal white adipose tissue and herein referred to as dWAT) is skin-associated adipose tissue. In rodents, dWAT is formed by a distinct layer of adipocytes residing directly below the reticular dermis, and is clearly separated from subcutaneous adipose tissue by a striated muscle layer, referred to as the panniculus carnosus, while in humans, no such structure exists (4). However, 2 distinct adipose layers have been defined based on the anatomical and metabolic heterogeneity of skin-associated adipose tissue in humans (5-8). Compared with other welldefined fat depots, murine dWAT is unique in its ability to expand and contract in response to various stimuli. During hair growth, hair follicle cycling can be divided into 3 main phases: anagen (growth), catagen (regression), and telogen (rest). Dermal adipocytes increase in size and number as hair follicles grow, and significantly decrease in volume as hair follicles regress. The synchrony of those 2 processes suggests a long-range interaction between hair follicles and dWAT (8-10). High-fat diet (HFD) and local infections can also efficiently induce the expansion of the dermal adipose layer (11, 12). During the regression phase of hair cycling (in catagen), dWAT undergoes a dramatic decrease in volume, but no obvious apoptotic events can be detected $(10,13)$. Currently, the oscillations of murine dWAT are thought to be controlled by changes in lipogenesis versus lipolysis similar to what is occurring in classical fat depots, although the molecular mechanisms regulating these dynamic changes are still poorly understood (14-16). Autophagy, another process capable of regulating lipid balance in adipocytes, can also be observed in dermal adipocytes during the regression of $\operatorname{dWAT}(17,18)$. 
Now it is well established that skin adipocyte precursors give rise to mature dermal adipocytes in the first wave of adipogenesis $(15,16)$. However, the fate of the mature dermal adipocytes during hair cycling and the origin of the rapidly reappearing dermal adipocytes in later waves of cycling remain unclear. Previous reports showed that mature adipocytes have the ability to dedifferentiate into multipotent cells in vitro $(19,20)$. In particular, recent studies in our laboratory revealed that adipocytes in the mammary gland display a remarkable level of plasticity in reversible dedifferentiation during lactation (21). Given the dynamic nature of adipogenesis in the skin, dermal adipocytes may have the potential to undergo dedifferentiation and transform to fibroblast-like cells.

In addition, a plethora of recent studies demonstrate that dWAT is involved in a wide range of processes, including hair cycling (15), local infections (12), and wound healing $(22,23)$. Owing to the challenges of isolating pure dWAT, this fat depot remains poorly characterized. At the same time, in light of the lack of animal models that specifically allow us to manipulate dWAT, the roles of mature dermal adipocytes in skin homeostasis remain ill-defined $(24,25)$. Here, we report the isolation and characterization of mature dermal adipocytes and define the structure as a unique fat depot capable of reversible de- and redifferentiation, thereby contributing to the dynamic nature of dWAT in adult mice. Through the use of a number of rodent models, we are highlighting an important role of mature dermal adipocytes in hair cycling and skin wound healing in mice. So far, there are limited insights into the status of human dWAT, though the heterogeneity of human skin-associated fat has been reported. Despite some anatomical differences between human and mouse skin, growing hair follicles, especially the scalp hair follicles, also root in skinassociated adipose tissue in humans, and a reduction of dWAT can be observed in patients with skin-related diseases (26-28). The skin-association of dWAT also indicates a role of human dWAT in skin function and skin diseases. Overall, this study advances our knowledge of dWAT and highlights the dermal adipocytes as an excellent target for interventions aimed at restoring healthy skin morphology and function.

\section{Results}

Isolation and characterization of dermal adipocytes. In mice, hair growth is highly synchronized for the first 2 cycles, hence the simultaneous oscillation of dWAT throughout the skin. Taking advantage of the anatomical location of dWAT and the unique properties of mature adipocytes, we developed a purification protocol for mature dermal adipocytes (Figure 1, A and B; and Supplemental Figure 1A; supplemental material available online with this article; https://doi.org/10.1172/JCI130239DS1). To our knowledge, this is the first protocol for dermal fat isolation in a murine model. We subsequently performed RNA-Seq on these highly purified dermal adipocytes and compared their gene expression patterns with those of purified adipocytes isolated from the subcutaneous layer. Although adjacent to each other, hierarchical clustering and gene ontology analysis revealed significant differences between these 2 types of adipocytes. We found that genes related to immune responses/inflammation were expressed at markedly higher levels, while genes related to cell adhesion and cell migration were expressed at much lower levels in dermal adipocytes compared with inguinal adipocytes (Supplemental Figure 1, B-D). As skin serves as the first antimicrobial barrier, the high expression of immune/inflammation response genes suggests a prominent role of dermal fat tissue in host defense. Differential gene expression analysis showed that dermal adipocytes expressed low levels of brown markers, such as Ucp1 and Cidea (Figure 1C). To further characterize dermal adipocytes, we compared their gene expression patterns with those of inguinal and gonadal adipocytes by quantitative PCR. General adipocyte marker genes were expressed at comparable levels in these 3 subtypes of adipocytes (Figure 1D and Supplemental Table 1). In agreement with the RNA-Seq data and the unilocular morphology that these cells display, we observed that dermal adipocytes expressed very low levels of marker genes characteristic of brown adipocytes. This puts dWAT clearly into the category of a white fat depot (Figure 1E). Nevertheless, unlike in gonadal adipocytes, Wt1, a marker gene for the gonadal fat depot, was not detected in dermal adipocytes (Figure 1F), highlighting the distinct nature of dWAT compared with gonadal fat. Consistent with their overall white adipocyte signature, dermal adipocytes displayed relatively low metabolic activity (Figure 1G). Furthermore, we screened the expression patterns of the 50 most upregulated genes in dermal adipocytes, and found that cathelicidin antimicrobial peptide (Camp) and chemokine (C-C motif) ligand 4 (Ccl4) were most abundantly expressed in dermal adipocytes compared with any other type of adipocyte (Figure 1, H and I, and Supplemental Figure 1, E and F). As inguinal adipocytes were found to also express significant levels of $\mathrm{Ccl} 4$ (Supplemental Figure 1E), we designated the Camp as the most specific marker to distinguish dermal adipocytes from any other type of adipocytes. In light of this unique Camp expression pattern and additional differential gene expression data, we can define dWAT as a unique fat depot with a clearly distinct identity.

Transcriptional profiling of dermal adipocytes during hair cycling. In murine skin, dWAT undergoes rapid and striking changes in synchrony with hair cycling. However, the underlying mechanisms remains poorly understood. To reveal the changes at transcriptome levels, we isolated mature dermal adipocytes from P21 (late catagen with thin dWAT), P25 (early anagen, dWAT starts to expand), P29 (middle anagen with thick dWAT), P35 (anagen, dWAT starts to regress), and P49 (telogen with thin dWAT), and performed RNA-Seq analysis with these samples. Venn diagram analysis showed that 10,788 genes were identified in all 5 groups. The numbers of differentially expressed genes are also indicated (Figure 2A). Principal component analysis demonstrated that each group of adipocytes exhibited a distinct transcriptome signature. The P21 and P49 groups were clustered much more closely relative to other groups, consistent with the timeline of the hair cycling (Figure 2B). We performed hierarchical clustering and gene ontology analysis to identify genes that change in a time course manner. We observed that genes involved in generation of metabolites and energy, electron transport chain, translation, and oxidation were enriched in anagen (P25, P29, P30) relative to catagen (P21) and telogen (P49). On the other hand, genes related to transcription, vascular development/blood vessel development/morphogenesis, cell proliferation, and general development showed opposite expression patterns (Figure 2, C-E). In agreement with the morphology of dermal adipocytes, genes related to adipogenesis were 
A

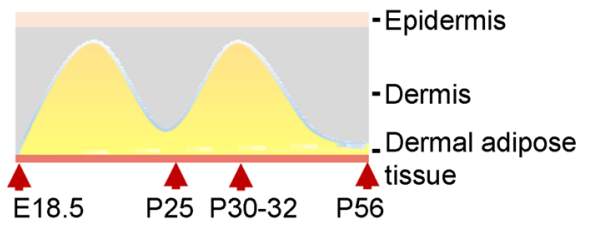

B

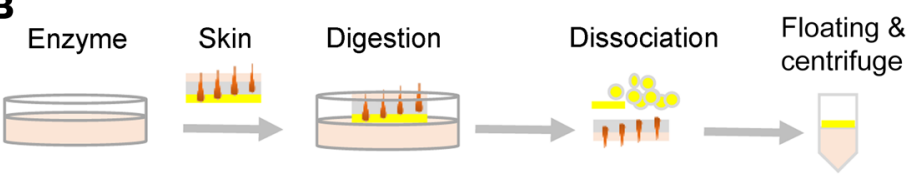

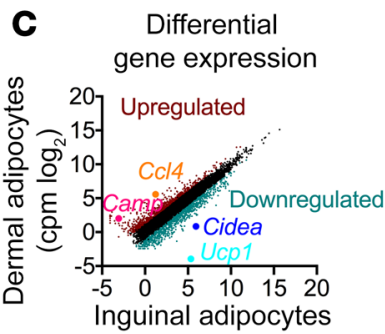

$\left(\mathrm{cpm} \log _{2}\right)$

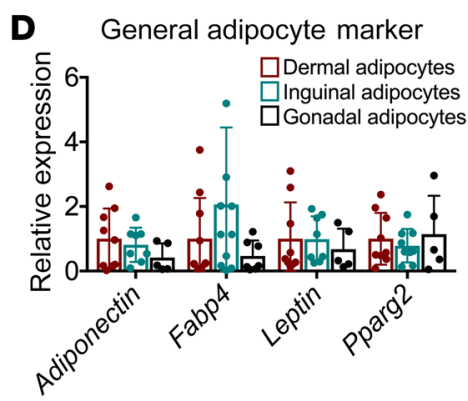

H

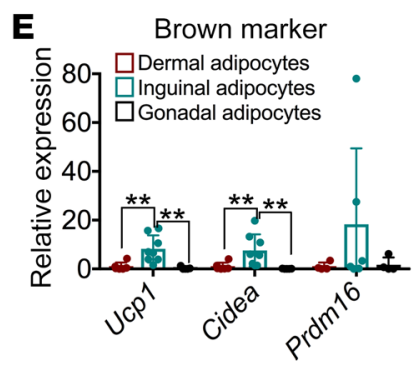

Camp 2-month-old

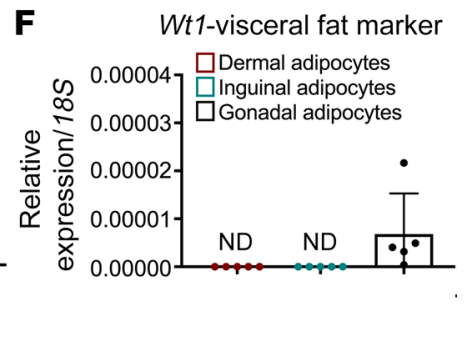

G

Metabolites

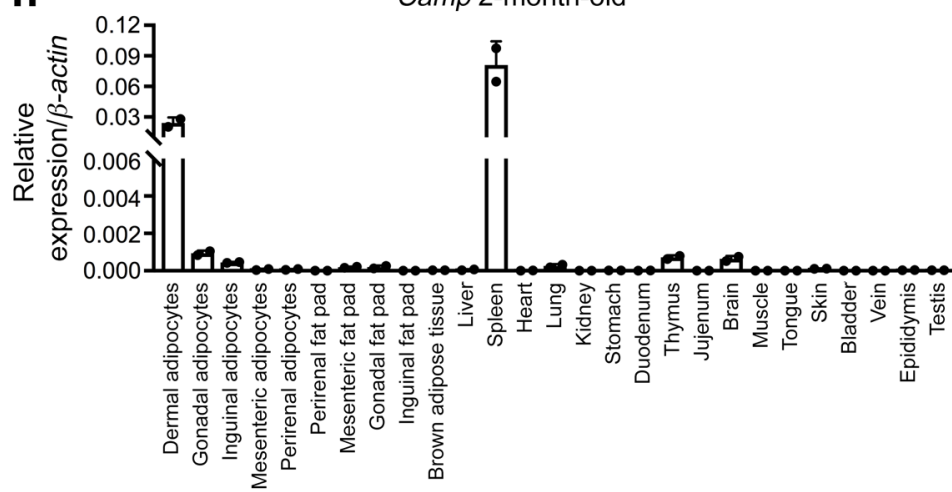

I Camp

30-day-old

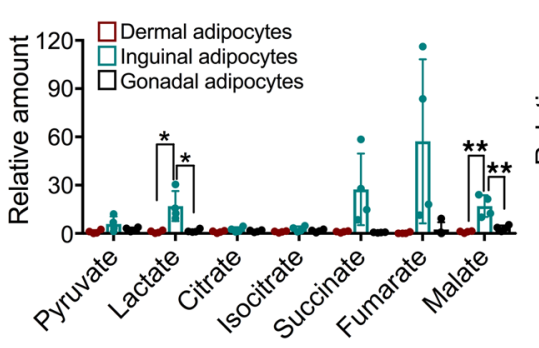

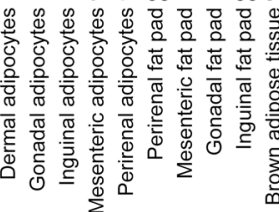

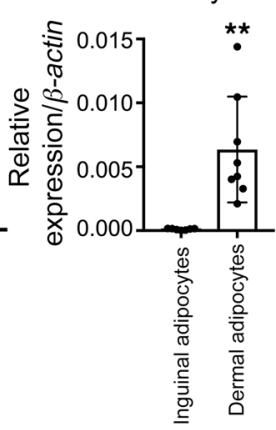

Figure 1. dWAT is a fat depot with a unique identity. (A) Schematic diagram of dWAT oscillation in mouse skin. (B) Graphic illustration of isolation of dermal adipocytes with floating digestion and floating centrifuge. (C) Differential gene expression analysis of the RNA-Seq results. Genes that changed less than 2-fold are represented with black dots. cpm, counts per million. (D-F) Reverse transcriptase PCR (RT-PCR) measurements of mRNA levels of general adipocyte marker genes (D), brown marker genes (E), and the visceral fat marker Wt1 (F) in dermal, inguinal, and gonadal adipocytes ( $n=5-10)$. ND, not detected. (C) Liquid chromatography-tandem mass spectrometry analysis of indicated metabolites extracted from dermal, inguinal, and gonadal adipocytes ( $n=4$ samples; each sample contained adipocytes from 3-4 mice). (H) Expression profile of Camp in different adipocytes, fat pads, and tissues in 2-month-old mice $(n=2)$. (I) Camp gene expression in 30-day-old mice $(n=6-7$, each sample pooled from 3-4 mice). All results were confirmed in at least 2 independent experiments. Data are shown as mean \pm SD. $P$ values were calculated with 2-tailed Student's $t$ test (I) or 2-way ANOVA with Tukey's test (D, $\mathbf{E}$, and $\mathbf{G})$. A $P$ value less than 0.05 is considered significant. ${ }^{*} P<0.05,{ }^{*} P<0.01$.

abundant in the anagen phase in comparison with catagen and telogen (Figure 2F). As anticipated, the positive correlation of Camp and dermal adipocyte differentiation was further confirmed in this system (Figure 2G). Wnt signaling, which inhibits adipocyte differentiation (29), was downregulated during the expansion of dermal adipocytes (anagen) (Figure 2H). In addition, lipolysis- and oxidation-related genes also showed increased levels during anagen (Figure 2I). Notably, we found that $B m p 1, B m p 2, B m p 4, B m p 6$, and $B m p 7$ were inhibited during anagen, while their antagonist, Noggin, was upregulated (Figure 2J). This was consistent with the previous study revealing that Bmp2 and Bmp4 inhibited stem cell activation during hair regeneration (30). Considering the ample amount of collagens in adipocytes, we checked the expression of collagen genes highly expressed in dermal adipocytes. Col4a1, Col4a2, Col5a3, Col6a2, and Col15a1 were significantly upregulated during anagen. Other collagens displayed distinct changes, but most of them showed reduced expression at the telogen phase (Figure 2K).
The differential expression of collagen genes suggests that dermal adipocytes may influence the structure of dermis during hair cycling. Thus, our data revealed the transcriptional changes of dermal adipocytes during hair cycling, which will be highly valuable for further mechanistic studies of dWAT and hair cycling.

The responses of dermal adipose tissue to classical changes that affect adipocytes. It is widely recognized that dWAT shows high plasticity in synchrony with hair cycling $(8,13,15)$. Recent reports described the expansion of the dermal adipose layer in parallel with body weight gain in obese mice exposed to either an HFD or leptin deficiency (11). These data were also confirmed by our own observations (Supplemental Figure 2, A-D). To investigate the maximal expansion capacity of dermal adipocytes, we assessed the thickness of dWAT in the most obese mice ever reported (31). The dermal fat layer in Ob/Ob-MitoNEET-Tg mice was about 3 times thicker than the layer seen in obese $\mathrm{Ob} / \mathrm{Ob}$ mice. On average, the thickness of the dWAT layer was more than $1 \mathrm{~mm}$, and com- 


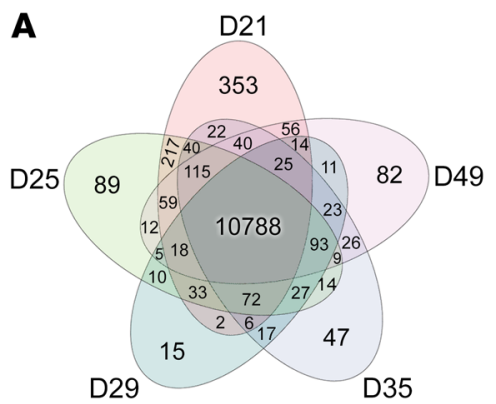

D

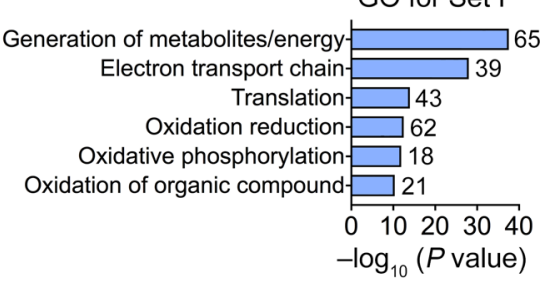

B

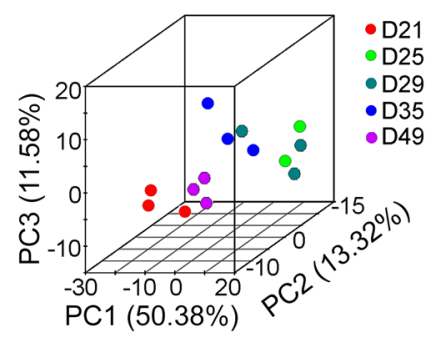

E

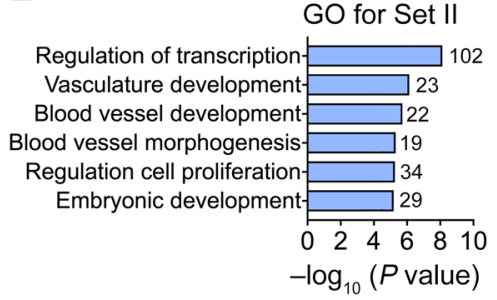

C

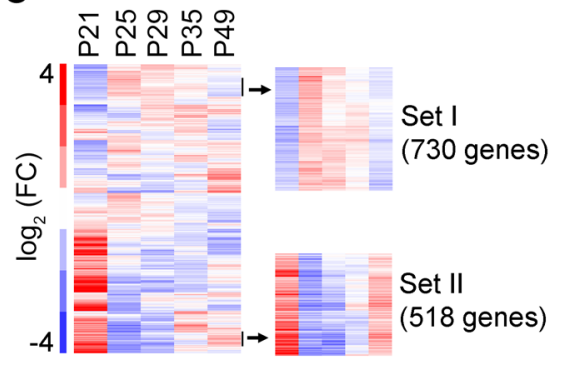

$\mathbf{F}$

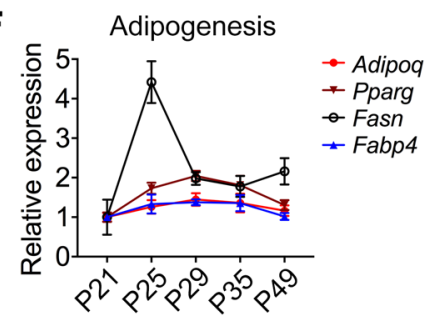

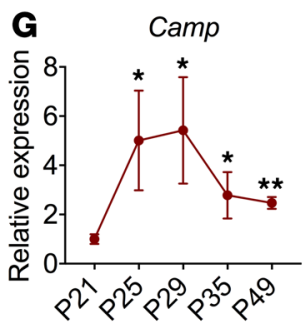
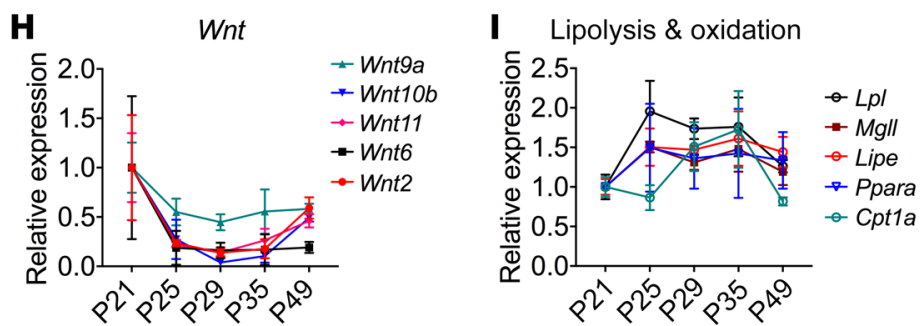

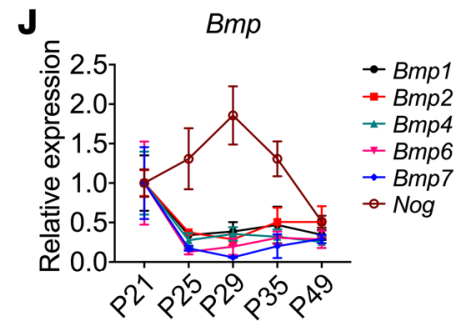

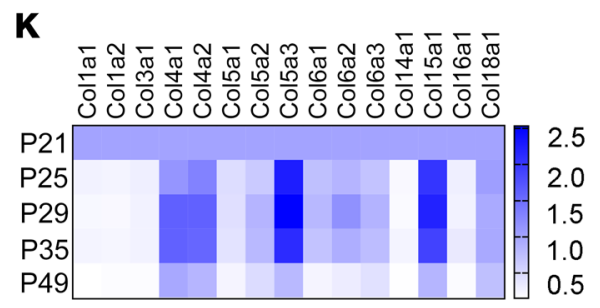

Figure 2. Transcriptional profiling of dWAT during hair cycling. (A) Venn diagram of genes expressed in dermal adipocytes harvested from 21-, 25-, 29-, 35-, and 49-day-old male mice, referred to as P21, P25, P29, P35, and P49. The coexpressed or uniquely expressed genes are visually presented. Default threshold of FPKM value for RNA-Seq is set to 1. Each group contained 3 samples; each sample contained dermal adipocytes harvested from 6 mice. (B) Principal component analysis of transcriptome of all samples. For all the data, 1 sample from D25 was excluded as it was clustered between P21 and P25. (C) Hierarchical clustering of transcriptional profile of P21, P25, P29, P35, and P49 dermal adipocytes. One cluster with enriched genes in anagen relative to catagen and telogen was defined as set I; one cluster with decreased genes in anagen relative to catagen and telogen was defined as set II. FC, fold change. ( $\mathbf{D}$ and $\mathbf{E}$ ) Gene ontology (GO) analysis of gene set I and set II. (F-J) Expression level of genes related to adipogenesis (F), Camp gene (G), Wnt family members (H), genes related to lipolysis and oxidation (I), Bmp family members, and Noggin (J) in dermal adipocytes during hair cycling. (K) Heatmap of collagen gene expression during hair cycling. One-way ANOVA with Dunnett's test. A $P$ value less than 0.05 is considered significant. ${ }^{*} P<0.05$, ${ }^{*} P<0.01$.

prised about 15 adipocytes along the vertical axis (Figure 3, A and B). It seems that both hypertrophy and hyperplasia are involved in the expansion of dWAT in these mice (Figure 3A and Supplemental Figure 2, A-D). In view of the special location of dWAT, topical application of rosiglitazone, a PPAR $\gamma$ agonist, also induced the expansion of dWAT (Figure 3, C and D). When we account for the surface area of the skin, the volume of dWAT is considerable in obese mice. In line with its overall white fat depot signature, dWAT showed robust resistance to cold-induced beiging (Supplemental Figure 2, E and F). Even with $\beta_{3}$-adrenergic agonist (CL-316,243) stimulation, no obvious multilocular beiging could be observed in the dWAT structure, while striking increases in multilocular cells were observed in subcutaneous fat (Figure 3, E and F, and Supplemental Figure 2G). This result further highlights the difference between dWAT and subcutaneous fat, and also indicates that dWAT does not mediate cold-induced thermogenesis, despite its more superficial anatomical location compared with subcutaneous fat. Furthermore, loss of dWAT is a hallmark of skin fibrosis $(4,23)$. In a bleomycin-induced skin fibrosis model, dermal adipocytes were barely detectable in the fibrotic skin (Supplemental Figure 2, $\mathrm{H}$ and I), reflecting a likely role of dWAT in skin fibrosis. Lipolytic action in the adipocytes has been implicated in the regression of dWAT (14). Phosphorylated residue serine in position 660 in hormone-sensitive lipase $\left(\mathrm{pHSL}^{\mathrm{S} 660}\right.$ ) can serve as a lipolysis marker 

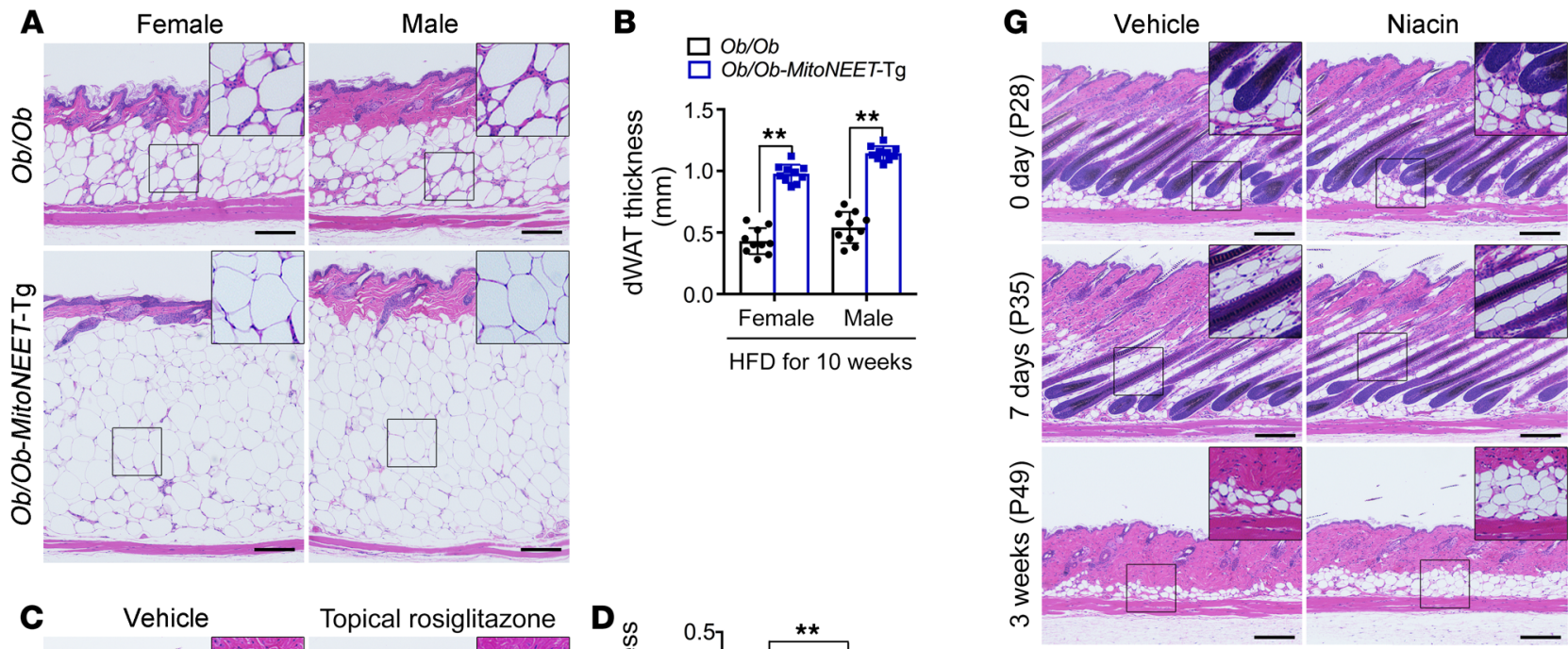

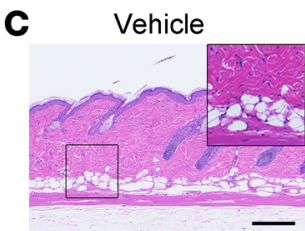

Topical rosiglitazone
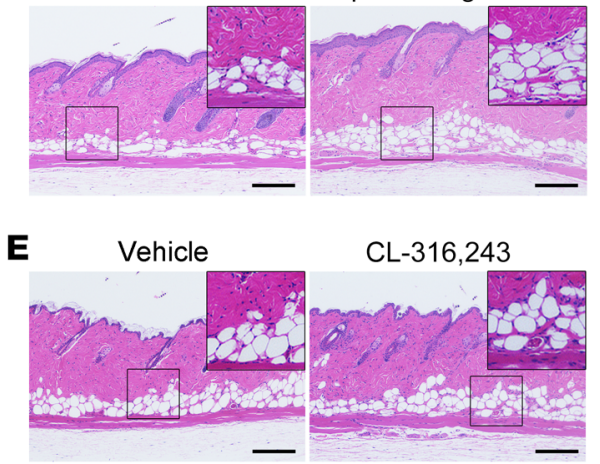
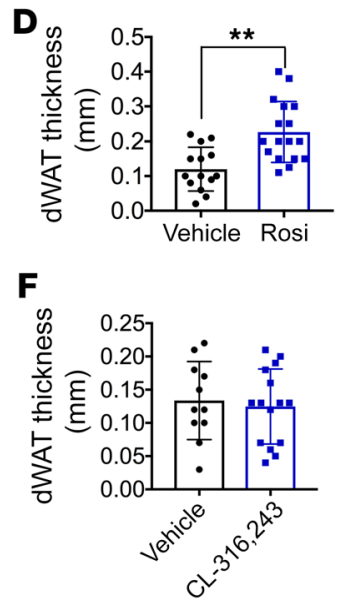

H

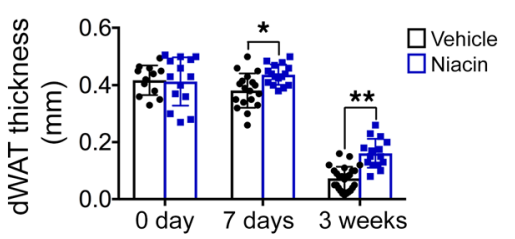

|
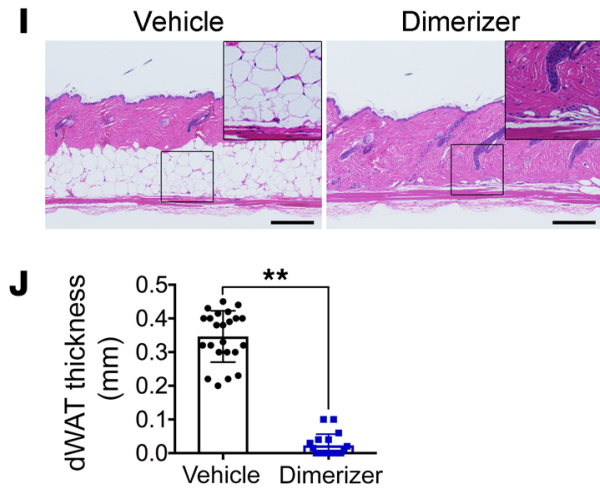

Figure 3. The responses of dWAT to different external challenges. (A) Histological sections (H\&E stained) of skin from Ob/Ob and $O b / O b-M i t o N E E T$-Tg mice with 10 weeks of HFD. Ob/Ob-MitoNEET-Tg mice showed a much thicker dWAT layer than that of $0 b / O b$ mice regardless of their sex ( $n=3$ per group). (B) Quantification of the dWAT thickness in A. (C) H\&E staining of skin from 2-month-old littermate mice treated topically with vehicle or rosiglitazone for 10 days. Topical application of rosiglitazone induced the local expansion of dWAT ( $n=5$ per group). (D) Quantification of the dWAT thickness in C. (E) No obvious multilocular cells were observed in the dWAT layer after 2 weeks of treatment with the $\beta_{3}$-adrenergic receptor agonist CL-316,243 ( $n=4$ per group). (F) Quantification of the dWAT thickness in E. (G) Twenty-eight-day-old mice were fed with niacin water for 1 and 3 weeks separately. The regression of dWAT was impaired with niacin treatment ( $n=5$ per group). (H) Quantification of the dWAT thickness in G. (I) Two weeks of dimerizer AP21087 injection nearly ablated dermal adipocytes in 1-year-old FAT-ATTAC mice $(n=5$ per group). (J) Quantification of the dWAT thickness in I. All results were confirmed in at least 2 independent experiments. Data are shown as mean \pm SD. $P$ values were calculated with 2-tailed Student's $t$ test (D, F, and J) or 2 -way ANOVA with Dunnett's test (B and $\mathbf{H}$ ). A $P$ value less than 0.05 is considered significant. ${ }^{*} P<0.05$, ${ }^{*} P<0.01$. Scale bars: $200 \mu \mathrm{m} ; \times 2$ magnification for insets.

and was detected in the dermal adipocytes (Supplemental Figure 2, $\mathrm{J}$ and $\mathrm{K})$. As expected, lipolysis inhibitors impeded the regression of dWAT (Figure 3, G and H, and Supplemental Figure 2, L-P). Specifically, in FAT-ATTAC mice (32), a model of inducible adipocyte cell death, dermal adipocytes underwent a very high degree of apoptosis following dimerizer injection that activated a caspase- 8 cassette, suggesting that this inducible lipodystrophy model is a good system to study dWAT biology (Figure 3, I and J). Taken together, these results show that dWAT displays dynamic changes under a variety of different conditions.
Dermal adipocytes arise from preexisting adipocytes during the expansion of dWAT in adult mice. To determine the fate of dermal adipocytes, we used AdipoChaser-mTmG mice, a doxycyclineinducible system for tracing of the fate of mature adipocytes (21, 33). As tdTomato is ubiquitously expressed in AdipoChaser-mTmG mice, we used perilipin 1 (PLIN1) staining instead of tdTomato to visualize adipocytes. Upon feeding of a doxycycline-containing diet, the existing adipocytes are labeled with green fluorescent protein (GFP) on the plasma membrane and appear as double-positive for GFP and PLIN1. After the labeling and washout periods 
A

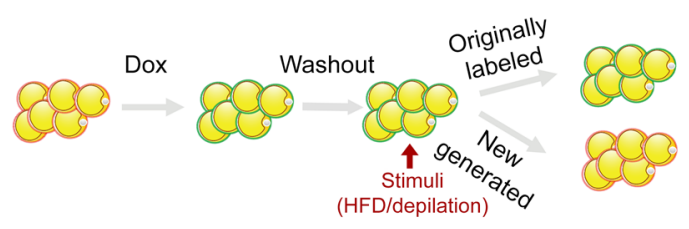

B

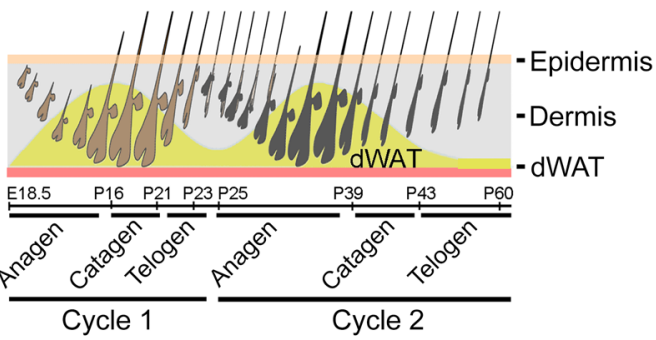

$\mathbf{F}$

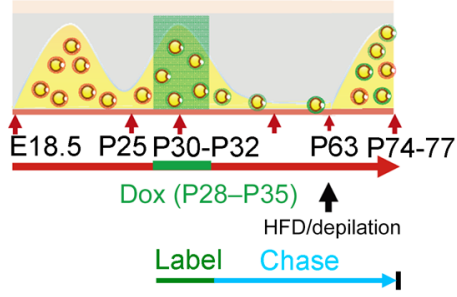

$\mathbf{G}$

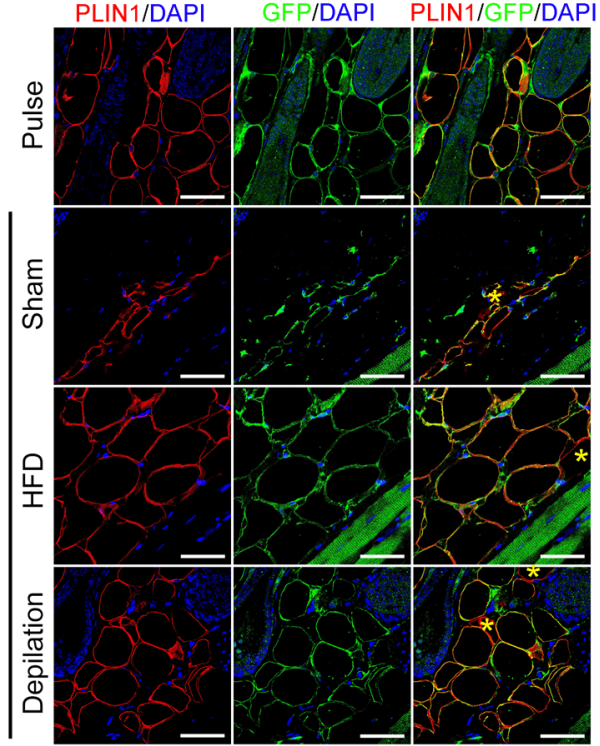

H

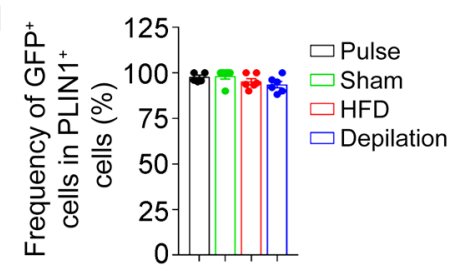

Figure 4. Lineage tracing of dermal adipocytes in vivo shows that a large number of dermal adipocytes arose from preexisting adipocytes during the expansion of dWAT in adult mice. (A) Schematic model for the labeling and tracing of dermal adipocytes in AdipoChaser-mTmG mice. (B) Hair follicle cycling and interactions with dWAT in male [57BL/6] mice. (C) Experimental design: labeling of the preexisting adipocytes at second telogen, when the dWAT had less mature adipocytes, and tracing of the adipocytes following HFD/depilation. (D) After doxycycline (Dox) chow diet (P56-P63), skins were harvested at P63 (Pulse). Thereafter, mice were fed with chow diet, and skin was harvested at P84 (Sham). Mice were fed with HFD (P70-P84) to induce the expansion of dWAT, and skin was harvested at P84 (HFD). Hair was depilated at P70 to induce the expansion of dWAT, and skin was harvested at P81P84 (Depilation). $n=5$ per group. (E) Quantification of the number of originally labeled adipocytes and new generated adipocytes in $\mathbf{D}$. (F) Experimental design: labeling of preexisting adipocytes at the second anagen, when dWAT layer has more mature adipocytes, and subsequent tracing of the adipocytes following HFD/depilation. (C) After Dox chow diet (P28-P35), skin was harvested at P35 (Pulse). Thereafter, mice were fed with chow diet, and skin was harvested at P77 (Sham). Mice were fed with HFD (P63-P77) to induce the expansion of the dWAT layer; skin was harvested at P77 (HFD). Hair was depilated at P63 to induce the expansion of dWAT, and skin was harvested at P74-P77 (Depilation). (H) Quantification of the number of originally labeled adipocytes and new generated adipocytes in $\mathbf{G} . n=4$ per group. Cells positive for only PLIN1 are marked with yellow asterisks. Data are shown as mean \pm SD. All results were confirmed in 3 independent experiments. Two-way ANOVA with Tukey's test. A $P$ value less than 0.05 is considered significant. ${ }^{* *} P<$ 0.01. Scale bars: $100 \mu \mathrm{m}$. 
to eliminate the doxycycline, the prelabeled adipocytes will still be GFP and PLIN1 double-positive, while newly generated adipocytes during the chase are GFP negative and appear as PLIN1 positive only (Figure 4A and Supplemental Figure 3A). Using this system, we traced the fate of dermal adipocytes in vivo. During the developmental stage, the first wave of dermal adipocytes is generated via proliferation and differentiation of precursor cells in synchrony with the first wave of hair follicle growth (first anagen) $(10,15)$. To determine whether de novo adipogenesis continues to the expansion of the dermal adipose layer during the second anagen phase, we labeled the preexisting adipocytes generated from the first anagen stage, and then tracked them to the second anagen (Figure 4B and Supplemental Figure 3B). Around $40 \%$ of adipocytes lacked a detectable GFP signal, suggesting a large amount of de novo adipogenesis during the second anagen. However, the percentage of newly generated adipocytes remained constant even under additional stimuli, such as exposure to an HFD or depilation (Supplemental Figure 3, C and D). To further determine the contribution of de novo adipogenesis in adult mice, we labeled the preexisting adipocytes of the second telogen (the second resting stage of the hair growth cycle, with very thin dWAT layer), followed by HFD or depilation to give rise to an expansion of dWAT (Figure 4C). A striking number of dermal adipocytes emerged as PLIN1 positive only, which indicated a new wave of adipogenesis (Figure 4, D and E). However, when we labeled the preexisting adipocytes during the second anagen (the second growth stage of hair growth cycle, with maximum number of dermal adipocytes since the mice were born), rather than during the second telogen, and traced their fate in adult mice under stimuli of HFD or depilation (Figure 4F), unexpectedly, most cells emerging after the second telogen were still GFP and PLIN1 double-positive (Figure 4, G and H). This remained the case even after 2 rounds of regression of dWAT induced by repeated depilation (Supplemental Figure 3, E-G). This demonstrates that those GFP-positive cells arise from preexisting mature adipocytes from the second anagen phase. It is rather remarkable that the GFP-positive adipocytes accounted for $90 \%-95 \%$ of total dermal adipocytes (Figure $4, \mathrm{~F}-\mathrm{H})$. Since dermal adipocytes undergo a substantial number/ volume decrease during catagen (regression phase of hair follicles) and telogen (resting phase of hair follicles) (Supplemental Figure 2A, Supplemental Figure 4B, and ref. 16), the impressive reappearance of GFP-positive adipocytes indicates that dermal adipocytes do not commit to undergo apoptosis or necrosis during the regression of dWAT. This is consistent with previous studies that showed that no obvious apoptosis of adipocytes was observed during catagen and telogen phases (10). Meanwhile, to further understand the contribution of de novo adipogenesis for dWAT as a function of age in adult mice, we labeled the preexisting dermal adipocytes in the second anagen phase and traced them for as long as 6 months (Supplemental Figure 3H). Around 10\%-15\% of GFP-negative cells were found among PLIN1-positive adipocytes in the dWAT layer (Supplemental Figure 3, I and J), reflecting a rather low de novo adipogenesis/turnover of dermal adipocytes in adult mice. Therefore, de novo adipogenesis mainly contributes to the expansion of dWAT during early developmental stages, while the reappearance of the preexisting dermal adipocytes is the main contributor to the expansion of dWAT in adult mice. The large reappearance of the preexisting dermal adipocytes raises the possibility that a reversible dedifferentiation of adipocytes may occur during the oscillation of dWAT, similar to the process that we described in the mammary gland (21).

Dermal adipocytes dedifferentiate into fibroblast-like and adipocyte-derived preadipocytes in vivo. To determine whether dermal adipocytes undergo actual dedifferentiation or merely an extended lipolytic phase during the regression of dWAT, we labeled dermal adipocytes during the second anagen, and then isolated cells from skin at the prolonged second telogen when dWAT displayed the most striking regression. The dedifferentiated mammary adipocytes regained the expression of PDGFR $\alpha$ (21), a marker expressed only in adipocyte precursors and preadipocytes but not in mature adipocytes $(24,34)$. If this reflects full dedifferentiation of dermal adipocytes, signals for both GFP and PDGFR $\alpha$ are expected to be present, i.e., double-positive cells should be observed (Figure $5 \mathrm{~A})$. Indeed, FACS analysis revealed a population of CD31-CD45PDGFR $\alpha^{+} \mathrm{GFP}^{+}$cells in doxycycline-pulsed mice (Figure $5 \mathrm{~B}$ ). This was further confirmed by the presence of GFP-positive fibroblast-like cells in the dWAT layer (Supplemental Figure 4A). These results are consistent with a dedifferentiation of mature dermal adipocytes in vivo. To compare the difference between dedifferentiated and nondifferentiated cells, we purified the CD31-CD45PDGFR $\alpha^{+} \mathrm{GFP}^{-}$and $\mathrm{CD} 31^{-} \mathrm{CD} 45^{-} \mathrm{PDGFR}^{+} \mathrm{GFP}^{+}$cells from skin for single-cell RNA-Seq, with approximately 7000 cells per group. The sequencing data revealed that CD31-CD45-PDGFR $\alpha^{+}$ $\mathrm{GFP}^{+}$cells were highly similar to $\mathrm{CD} 31^{-} \mathrm{CD} 45^{-} \mathrm{PDGFR} \alpha^{+} \mathrm{GFP}^{-}$cells (Figure 5C). Two distinct subpopulations were identified in both populations, termed cluster 1 and cluster 2 (Supplemental Figure 4, B and F). Gene ontology analysis revealed that cluster 1 was enriched for genes related to extracellular matrix and redox homeostasis, while cluster 2 was enriched for genes related to translation and immune responses (Supplemental Figure 4, B-I, and Supplemental Figure 5D). Both clusters 1 and 2 displayed high expression of fibroblast markers. In particular, cluster 1 was also enriched for genes related to adipogenesis and mature adipocyte markers, such as Pparg, Fabp4, Lpl, and Plin2. However, compared with mature adipocytes, these markers were only expressed at very low levels. Nevertheless, this indicates that cluster 1 contained an adipogenic precursor population (adipocyte-derived preadipocytes [ADPs]) (Figure 5D). The overall gene expression pattern demonstrates that the dedifferentiated adipocytes lost their mature adipocyte characteristics for the most part and regained full fibroblast and preadipocyte signatures (Figure 5, $D-G)$. To further check the heterogeneity of these cells, unbiased cell clustering revealed a total of 15 clusters in the combined cell population (Figure $5 \mathrm{H}$ and Supplemental Figure 4J). Although no obvious difference was observed at transcriptome levels, cell cluster distribution analysis demonstrated that CD $31^{-} \mathrm{CD} 45^{-} \mathrm{PDGFR} \alpha^{+}$ $\mathrm{GFP}^{+}$cells were enriched in clusters 0 (inflammation/wounding response), 5 (lipid metabolism), and 12 (vascular development), and decreased in clusters 3 (cell adhesion), 6 (inflammation/ immune response), 8 (signaling transduction/cell communication), and 11 (cell adhesion/cytoskeleton) (Figure 5, I and J; Supplemental Figure 4K; and Supplemental Table 2). In addition, cell cycle analysis showed that more CD31-CD45-PDGFR $\alpha^{+} \mathrm{GFP}^{+}$cells were detected at $\mathrm{S}$ phase (Supplemental Figure 4, L and M, and 
A

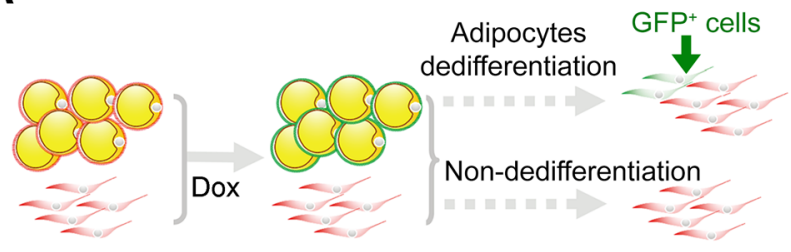

B Live gate: -Dox CD31-CD45-PDGFR $\alpha^{+}$

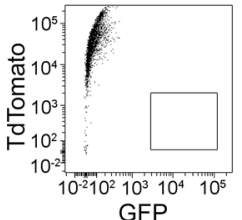

Live gate: +Dox CD31-CD45-PDGFR $\alpha^{+}$

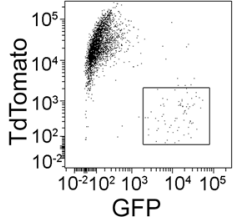

C

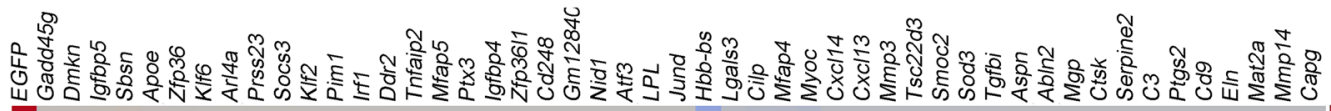

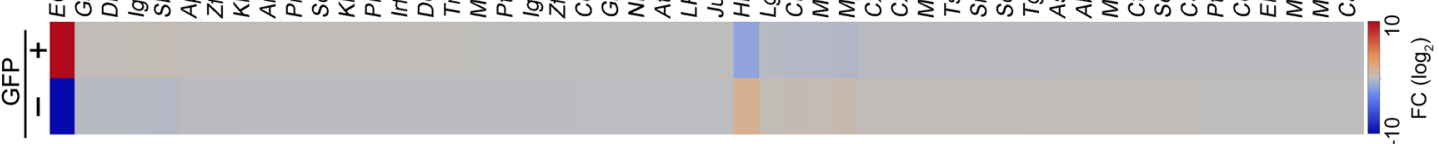

D $\frac{\text { Fibroblast marker }}{\text { GFP }^{+}}$
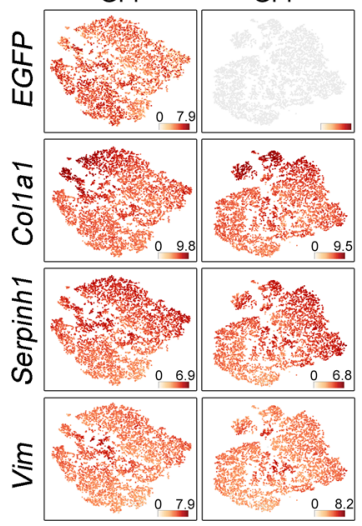

E

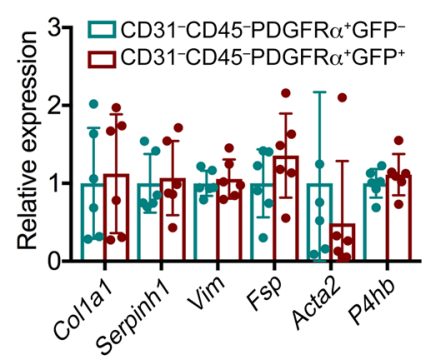

$\mathbf{H}$

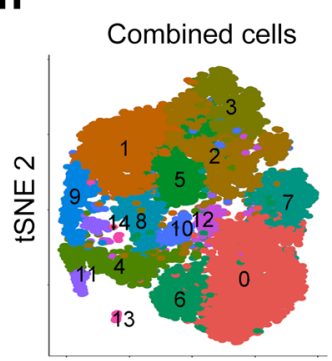

tSNE 1
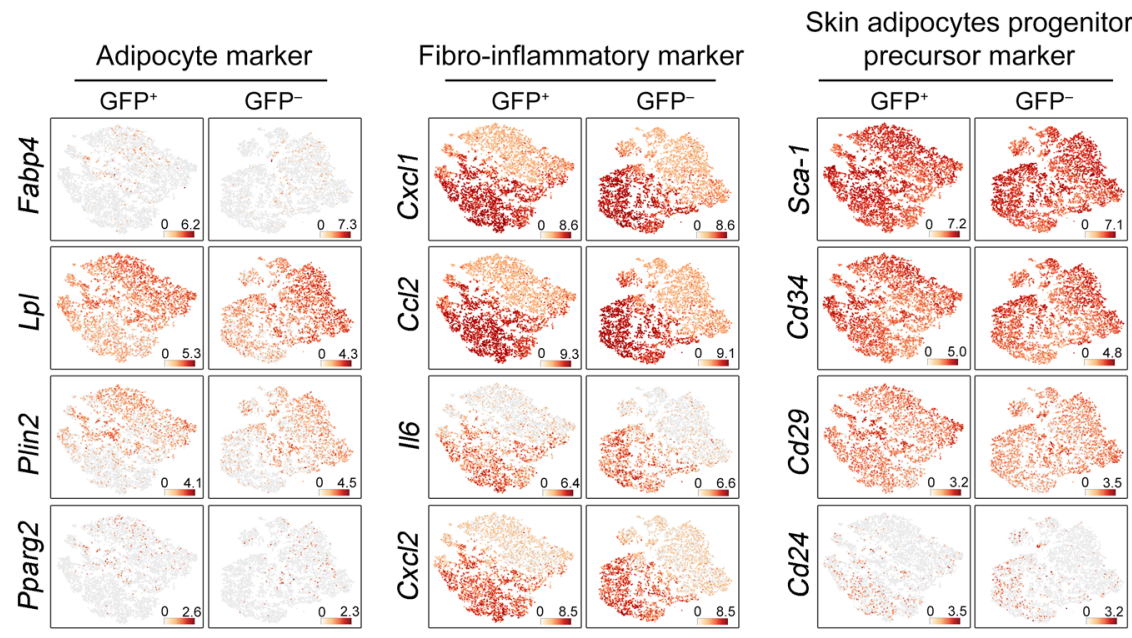

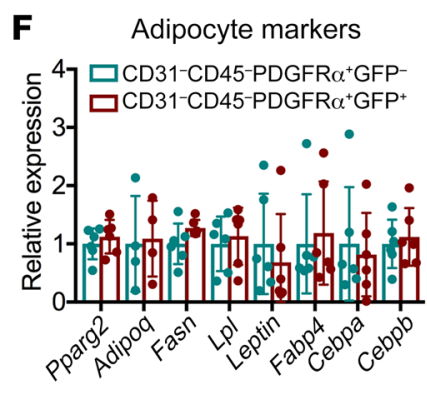

I

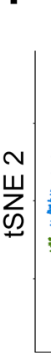

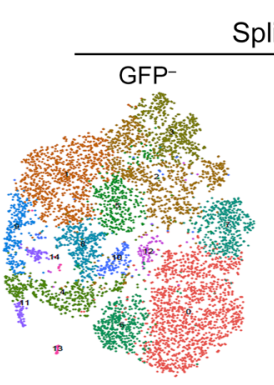

tSNE 1
Split

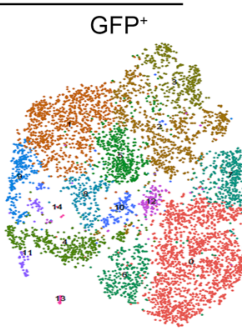

tSNE 1
G Stem cell markers

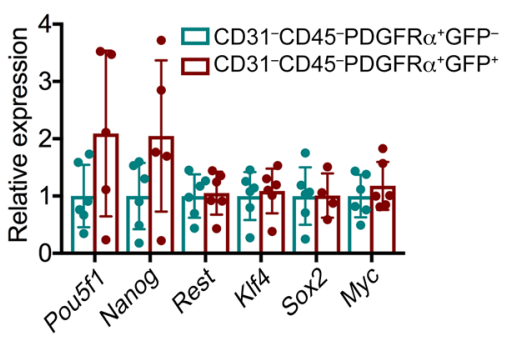

J Number of cells in each cluster

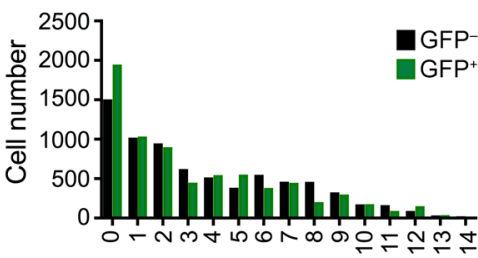
Cluster 
Figure 5. Characterization of dedifferentiated dermal adipocytes by single-cell RNA-Seq. (A) Schematic diagram of the dedifferentiation of dermal adipocytes in AdipoChaser-mTmG mice. In the presence of Dox, adipocytes were indelibly labeled with membrane-bound GFP. In the case of dedifferentiation of dermal adipocytes, the dedifferentiated cells will be GFP positive. (B) The gating strategy showed the presence of CD31-CD45-PDGFR $\alpha^{+}{ }^{-}$FP $^{+}$cells in skin of Dox-treated AdipoChaser$\mathrm{mTmG}$ mice ( $n=3$ per group). Results were confirmed by 2 independent experiments. (C) Heatmap of the top 25 most differentially expressed genes between CD31-CD45-PDCFR $\alpha^{+}$GFP- and CD31-CD45-PDCFR $\alpha^{+}{ }^{+}$GP $^{+}$ cells. (D) Gene expression pattern of fibroblast marker genes, common adipocyte marker genes, fibrosis and inflammation marker genes, and skin adipocyte progenitor/precursor marker genes as displayed by a t-distributed stochastic neighbor embedding (tSNE) plot. Transcript counts represent $\log _{2}$ of gene expression. Plots were made using the Loupe Cell Browser (10x Genomics). (E-G) RT-PCR measurements of mRNA levels of fibroblast marker genes, common adipocyte marker genes, and stem cell marker genes in purified CD31-CD45-PDGFR $\alpha^{+} \mathrm{CFP}^{-}$and $\mathrm{CD}^{-}$- $^{-}$CD45-PDGFR $\alpha^{+} \mathrm{CFP}^{+}$ cells. RT-PCR results were confirmed by 3 independent experiments. $n=$ 5-6 mice. (H) Unbiased clustering of combined cells reflects 15 clusters. (I) Split view of the CD31-CD45-PDCFR $\alpha^{+}$CFP $^{-}$and CD31-CD45-PDCFR $\alpha^{+}{ }^{+}{ }^{-}{ }^{-}{ }^{+}$ cells in $\mathbf{H . ~ ( J ) ~ Q u a n t i f i c a t i o n ~ o f ~ t h e ~ G F P - ~ a n d ~ G F P + ~ c e l l s ~ i n ~ e a c h ~ c l u s t e r . ~ D a t a ~}$ are shown as mean $\pm \mathrm{SD}$. $P$ values were calculated with 2 -way ANOVA with Dunnett's test. A $P$ value less than 0.05 is considered significant.

Supplemental Table 3), which indicated that some of them might be more prepared for proliferation. Therefore, our data reveal widespread dedifferentiation of mature dermal adipocytes. The differentiated $\mathrm{CD} 31^{-} \mathrm{CD} 45^{-} \mathrm{PDGFR} \alpha^{+} \mathrm{GFP}^{+}$cells are highly similar to $\mathrm{CD} 31^{-} \mathrm{CD} 45^{-} \mathrm{PDGFR} \alpha^{+} \mathrm{GFP}^{-}$cells, but differences are apparent in cell cluster distribution and cell cycle progression.

Dedifferentiated dermal adipocytes exhibit potential for proliferation and can transform into myofibroblasts. Given our finding that dermal adipocytes can dedifferentiate into fibroblast-like and ADP-like cells, we tested whether these dedifferentiated adipocytes had the potential to proliferate or transdifferentiate into other cell types in vivo. We labeled the preexisting dermal adipocytes during the second anagen when there was a maximum number of dermal adipocytes since the birth of the mouse, and then followed these cells to the second telogen when there was a very thin dWAT layer (with the highest level of dedifferentiation of dermal adipocytes since birth) (Figure 4B). To test the proliferative potential of the dedifferentiated adipocytes, mice were fed with an HFD at P56 (second telogen) to induce the expansion of dWAT, and BrdU was injected daily simultaneously. Within 1 week of HFD, BrdU-positive cells were observed in the expanding dWAT layer and costained with GFP, which suggested the proliferation of dedifferentiated adipocytes (Figure 6A). As expected, $\mathrm{CD}^{-} 1^{-} \mathrm{CD} 45^{-}{ }^{-}{ }^{-} \mathrm{CFR}^{+} \mathrm{GFP}^{-}$as well as CD $31^{-} \mathrm{CD} 45^{-} \mathrm{PDGFR}^{+} \mathrm{GFP}^{+}$ cells proliferated well in vitro (Supplemental Figure 5A). Interestingly, when we stimulated them for differentiation, the dedifferentiated $\mathrm{CD}^{-} 1^{-} \mathrm{CD} 45^{-} \mathrm{PDGFR} \alpha^{+} \mathrm{GFP}^{+}$cells displayed higher adipogenic potential than $\mathrm{CD}^{-} 1^{-} \mathrm{CD} 45^{-} \mathrm{PDGFR}^{+} \mathrm{GFP}^{-}$cells, as reflected by the number of adipocytes and the expression level of adipocyte marker genes (Supplemental Figure 5, B and C). To test the possibility of transdifferentiation of the dedifferentiated adipocytes, we took advantage of a bleomycin-induced fibrosis model, which is widely used to mimic early and inflammatory stages of systemic sclerosis (35). During the fibrotic process, skin fibroblasts not only secrete extracellular matrix, cytokines, and growth factors, but also can undergo transdifferentiation, and morph into myofibroblasts with unique expression of $\alpha$-smooth muscle actin ( $\alpha$-SMA) (36). Previous studies demonstrated that adiponectin-positive intradermal progenitors gave rise to dermal myofibroblasts (37). Here, we investigated whether the dedifferentiated dermal adipocytes have the capacity to morph into myofibroblasts under bleomycin exposure and whether this may be accompanied by the expected substantial increase in myofibroblast numbers (38). A population of $\alpha$-SMA-positive myofibroblasts was costained with GFP in the fibrotic dermis, whereas no obvious costaining was observed in the vehicle group (Figure 6B). As $\alpha$-SMA is a specific marker for myofibroblasts, this observation suggests that the dedifferentiated adipocytes have the capacity to convert to myofibroblasts in vivo. We then purified the CD31-CD45-PDGFR $\alpha^{+}$ $\mathrm{GFP}^{-}$and $\mathrm{CD}^{-} \mathrm{CD} 45^{-} \mathrm{PDGFR} \alpha^{+} \mathrm{GFP}^{+}$cells, and stimulated them with TGF- $\beta$, an inducer of myofibroblast differentiation. No differences in the expression levels of downstream target genes were detected (Supplemental Figure 5D), which indicates that the potential of the dedifferentiated CD31-CD45-PDGFR $\alpha^{+}$ $\mathrm{GFP}^{+}$cells to transform into myofibroblasts is comparable to that of $\mathrm{CD}^{-} 1^{-} \mathrm{CD} 45^{-} \mathrm{PDGFR}^{+} \mathrm{GFP}^{-}$cells. We conclude that the dedifferentiated dermal adipocytes not only regain the ability to proliferate and redifferentiate into adipocytes, but they also have the potential to transition into myofibroblasts in response to an appropriate stimulus, such as bleomycin.

Manipulating dermal adipocytes reveals their important roles in hair cycling and skin wound healing. During hair growth, hair follicles strongly interact with dermal adipocytes $(8,24)$, suggesting a role of dWAT in hair cycling. Various correlations between hair cycling and dWAT have been observed in a number of mouse models $(15,24)$. However, most of the mouse models used in these studies are germline mutants, and do not specifically target adipocytes. Even PPAR $\gamma$, beyond its prominent expression in mature adipocytes, is also expressed in several additional cell types in the skin, such as matrix keratinocytes, dermal papilla, and sebocytes $(39,40)$. To specifically target adipocytes, we used the Adiponectin promoter-driven Cre mouse to enable an adipose-specific deletion of Pparg (Pparg ${ }^{A K O}$ ) without affecting Pparg in other cell types. We found that Pparg ${ }^{A K O}$ mice exhibited delayed formation of the hair coat (Supplemental Figure 6A), which was consistent with previous studies (41). Also, we observed that Pparg $^{A K O}$ mice developed severe fatty liver as early as 1 week of age, and these mice showed stunted growth compared with their littermates. Therefore, it is hard to distinguish the direct effects of dWAT from an overall delayed development (Supplemental Figure 6, A-F). In light of the essential role of lipids for early development, we used FAT-ATTAC mice (32), a model of inducible lipoatrophy, to ablate mature adipocytes at later stages. By targeting adipocyte ablation to a later point, any developmental deficiencies can be avoided. Initially, we started to induce the ablation of adipocytes at day P7. We found that dermal adipocytes were substantially reduced, while no obvious defect of hair cycling was observed during the second cycle of hair growth (Supplemental Figure 7A). In contrast to the findings in Pparg ${ }^{A K O}$ mice, ablation of adipocytes following depilation enhanced rather than inhibited the reentry into anagen in the depilated adult stage (Figure 7, A-E). In addition, we induced the expansion of dermal adipocytes by HFD in adult 
A

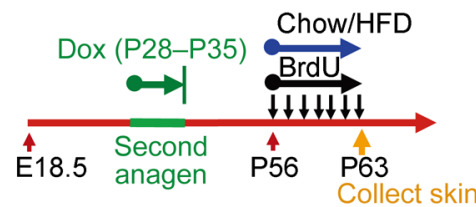

BrdU/DAPI

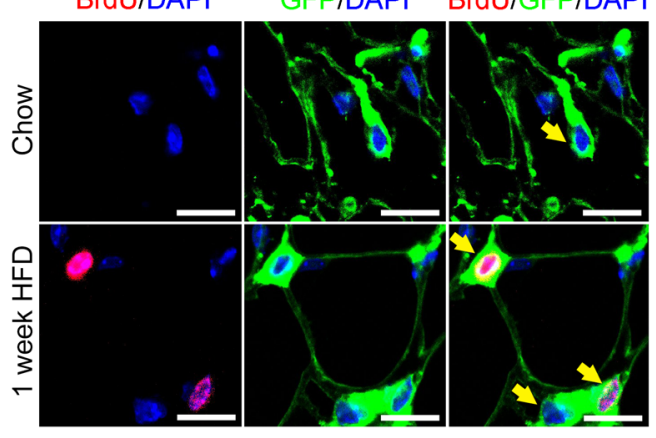

B

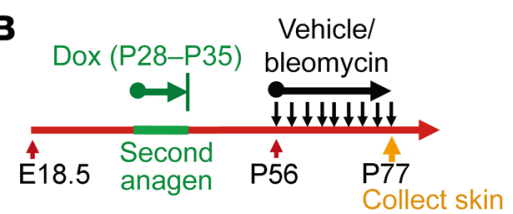

Vehicle

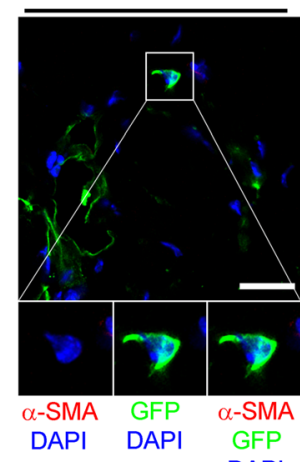

DAPI

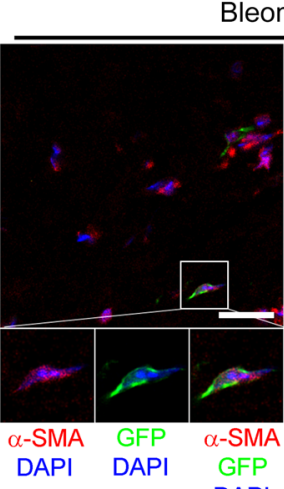

DAPI
Bleomycin

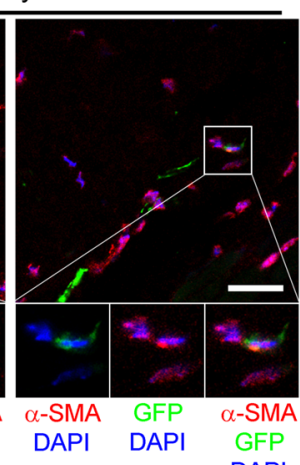

DAPI

Figure 6. In vivo detection of proliferation and transformation of dedifferentiated dermal adipocytes. (A) Schematic diagram of experimental design: Preexisting adipocytes were labeled during the second anagen in AdipoChaser-mTmG mice (Dox: P28-P35). The mice were then put on normal chow diet until a prolonged period into the second telogen. One group of mice was on normal chow diet and was injected with BrdU daily (P56-P63). Another group of mice was switched to HFD, and injected with BrdU daily (P56-P63). Skin was collected at P63 for GFP (green), BrdU (red), and DAPI (blue) staining. Colocalization of GFP and BrdU was observed in the HFD group, but not in the chow diet group. $n=3$ mice per group. (B) Schematic diagram of experimental design: Preexisting adipocytes were labeled during second anagen in AdipoChaser-mTmG mice (Dox: P28-P35). Then mice were put on normal chow diet until prolonged second telogen. From P56, mice were injected s.c. with vehicle/bleomycin daily for a total of 21 days. Skin was collected at P77 for GFP (green), $\alpha$-SMA (red), and DAPI (blue) staining. Colocalization of GFP and $\alpha$-SMA was observed in the bleomycin-treated group, but not in the vehicle group. $n=4$ mice per group. Results were confirmed in 3 independent experiments. Scale bars: $50 \mu \mathrm{m} ; \times 2$ magnification for insets.

mice, and then depilated the hair to induce hair cycling (Figure $7 F)$. Mice on an HFD displayed a significant delay in the reentry into the anagen phase (Figure 7, G-J). However, when we initiated the HFD from P7, no obvious impact on the second hair cycle was observed (Supplemental Figure 7, D and E). Nevertheless, the HFD group developed various levels of alopecia on the dorsal skin around weaning age, due to the hair shafts being broken in the hair follicles (Supplemental Figure 7, F and G), suggesting a potent role of dWAT in maintaining hair integrity. Notably, upon ablation of dermal adipocytes, the dermis became significantly thicker, probably reflecting a compensation for the loss of dermal adipocytes (Figure 7C and Figure 3I).

To address whether mature dermal adipocytes are required for wound healing in the skin, we assessed the recovery from a splinted wound in lipodystrophic FAT-ATTAC mice and their WT counterparts. Wound closure was assessed as a function of time. The healing process in the skin of FAT-ATTAC mice, compared with WT controls, was significantly impaired (Figure 7K and Supplemental Figure 7, H and I). Upon analysis of the proliferative capacity of keratinocytes, the FAT-ATTAC and the control mice had comparable levels of Ki67 $7^{+}$cells around the edge of the wound (Figure 7, L and M), reflecting unaltered proliferative capacity of the re-epithelializing keratinocytes. We subsequently assessed the migratory potential of the keratinocytes. FAT-ATTAC mice displayed normal migration capacity. However, the keratinocytes migrated along the wound edge instead of migrating to the wound bed (Figure 7N and Supplemental Figure 7I). Closer inspection of the wound bed showed that WT mice had a thicker wound bed, whereas the FAT-ATTAC mice had a thinner wound bed due to the lack of both subcutaneous fat and dermal fat (Supplemental Figure 7I). As adipocytes are also a rich source of extracellular matrix components, we checked the collagen deposition in the wound bed with trichrome staining, thereby highlighting collagen fibers in blue. Considerably less collagen was deposited, as reflected by the reduced blue stain observed (Figure 70). These results indicate that dermal adipocytes are required for the proper establishment of the wound bed during wound healing. However, there are no available mouse models that can specifically manipulate dermal adipocytes. During the wound healing process, subcutaneous adipose tissue was also recruited into the wound bed and temporally fused with the forming scar tissue (Supplemental Figure 7I). This is consistent with high expression of adhesionand migration-related genes in subcutaneous adipocytes (Supplemental Figure 1, B and D). Meanwhile, ablation of adipose tissue results in insulin resistance, which also contributes to the impaired wound healing. Nonetheless, mature dermal adipocytes are rich resources for adipokines and cytokines and also expressed higher levels of immune/inflammation response-related genes and collagen genes compared with subcutaneous adipocytes (Supplemental Figure 1, B and C, and Figure 7P). We checked databases with human gene expression data, and found that $A D I P O Q$ was significantly downregulated in lesional psoriasis skin biopsies compared with normal or nonlesional psoriasis individuals. The reduction of $A D I P O Q$ was also observed in human keloid biopsies. However, normal wound skin showed increased $A D I P O Q$ expression (Figure 7, Q and S, and Supplemental Figure 7, J and K). As cathelicidins have been reported to be directly involved in host defense and wound healing in both mice and humans (12, 42-44), 
A

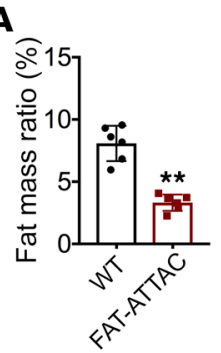

B

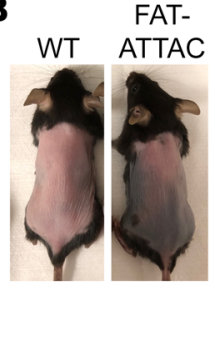

C

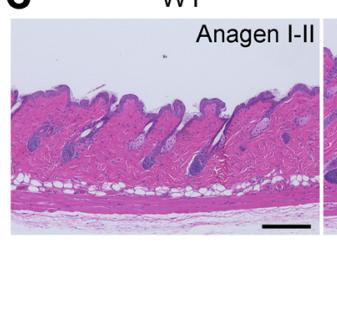

FAT-ATTAC

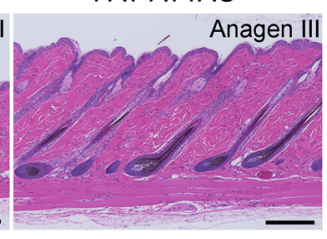

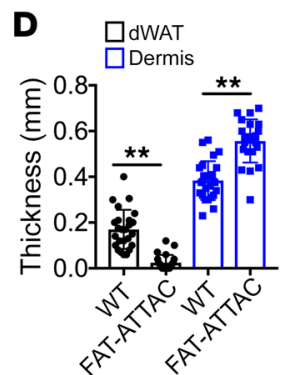
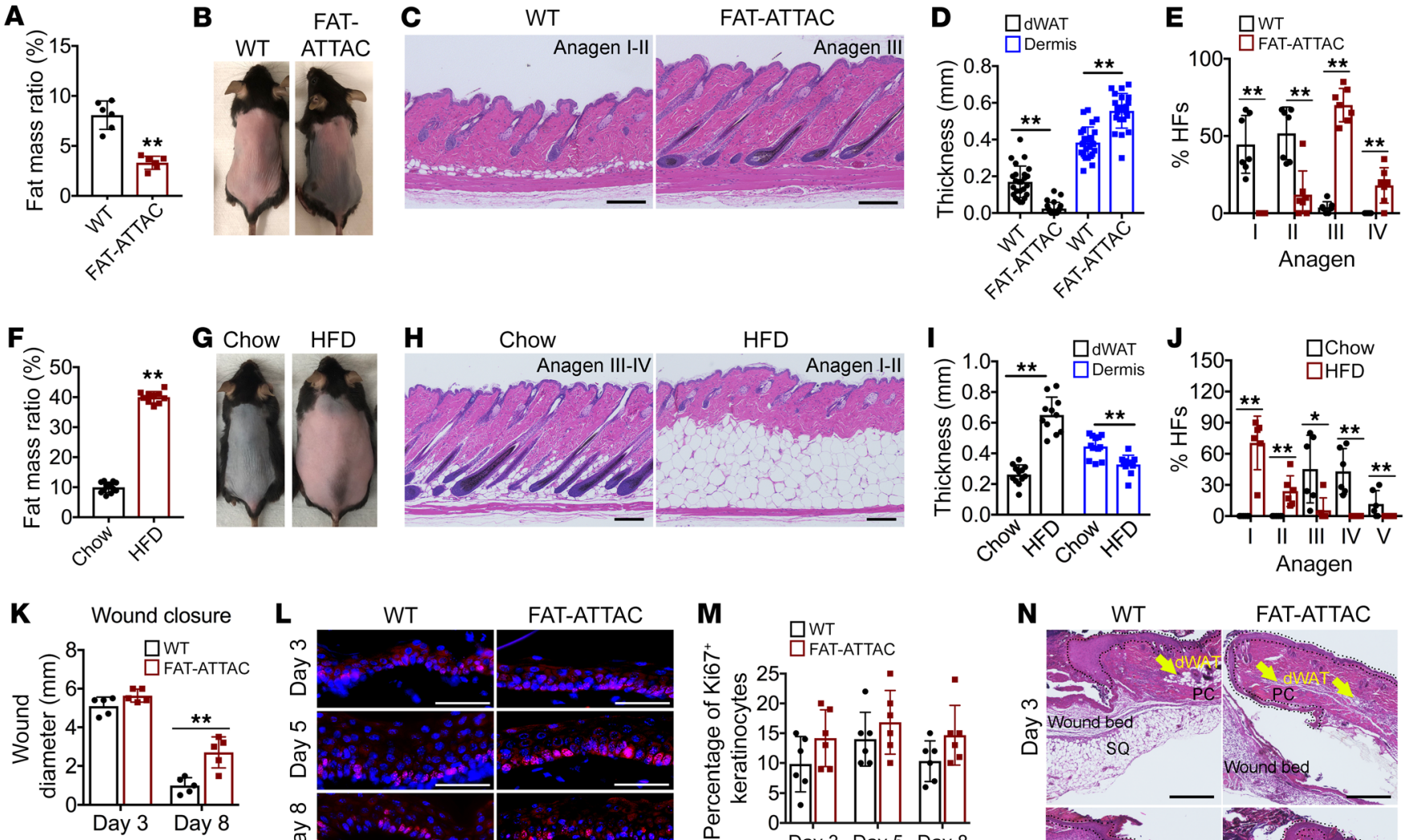
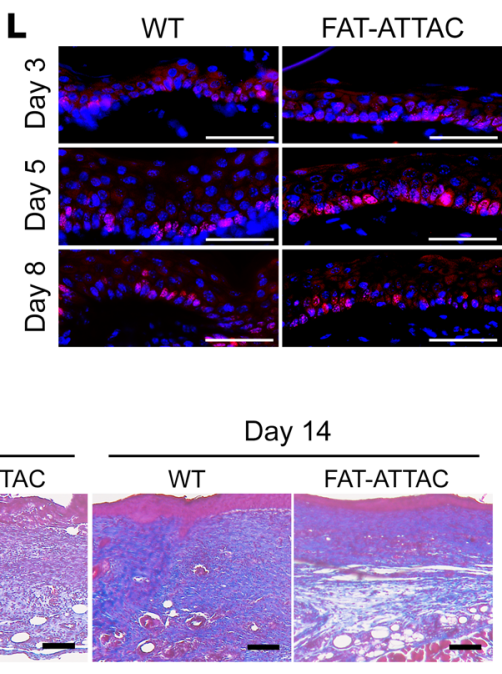
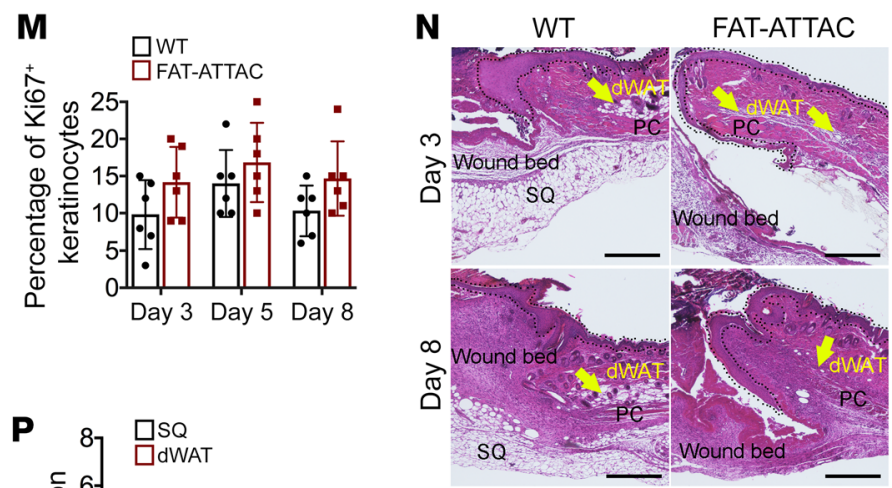

s

ADIPOQ in psoriasis skin (GSE13355)

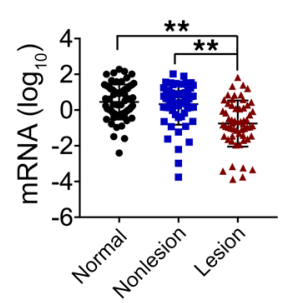

$\mathbf{R}$

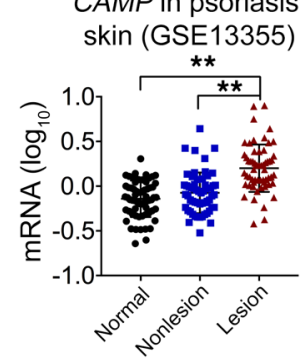
$A D I P O Q$ in psoriasis skin (GSE30999)

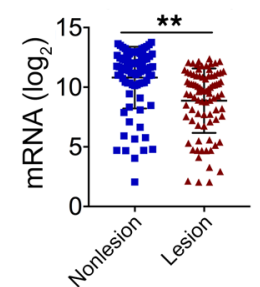

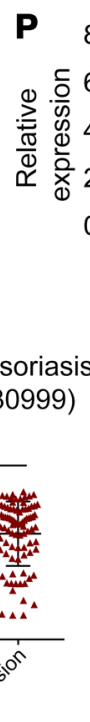

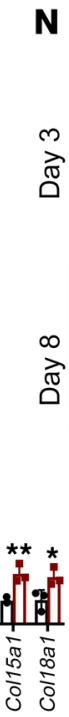

$\mathbf{T}$ CAMP in psoriasis skin (GSE30999)

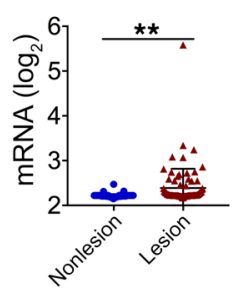

U

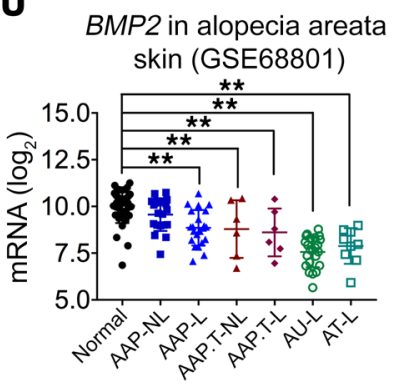

Figure 7. Mature dermal adipocytes are required for hair cycling and skin wound healing. (A) FAT-ATTAC mice showed reduced fat mass after dimerizer injection. (B) Depilation of hair to induce reentry of hair cycle in WT and FAT-ATTAC mice ( $n=7-10$ per group). (C) FAT-ATTAC mice showed faster reentry into anagen than littermate controls. Scale bars: $200 \mu \mathrm{m}$. (D and E) Quantification of dWAT/dermal thickness, and hair cycle stage of hair follicles in C. (F) HFD-treated mice showed significantly increased fat mass. (C) Depilation of hair to induce the hair cycling in chow/HFD-fed mice ( $n=6$ per group). (H) HFD-fed mice show impaired reentry into anagen compared with chow-fed controls. Scale bars: $200 \mu \mathrm{m}$. (I and J) Quantification of dWAT/dermal thickness, and hair cycle stage of hair follicles in H. (K) FAT-ATTAC mice show delayed wound closure ( $n=5$ per group). (L) Epithelial cell proliferation was assessed by Ki67 immunostaining. Scale bars: $50 \mu \mathrm{m}$. (M) Quantification of percentage of Ki67+ epithelial keratinocytes. (N) H\&E staining of wound edge. Epidermal layer located in area within the dotted line. Scale bars: $500 \mu \mathrm{m}$. PC, panniculus carnosus. (0) Collagen production is reduced in the wound gap of FAT-ATTAC mice compared with vehicle controls as judged by trichrome staining (collagen: blue). Scale bars: $200 \mu \mathrm{m}$. (P) RT-PCR measurements of mRNA levels of collagen-related gene expression in isolated subcutaneous and dermal adipocytes of WT mice ( $n=3$ per group). SQ, subcutaneous adipose. (Q-T) ADIPOQ and CAMP expression in skin biopsies from 64 healthy controls and psoriatic patients. One lesion and one nonlesion biopsy from each patient; 58 patients for GSE13355, 85 patients for GSE30999. (U) BMP2 expression in scalp skin biopsies from 36 healthy controls and alopecia areata patients (AAP). NL, nonlesion; L, lesion; T, transit; AU, alopecia universalis; AT, alopecia totalis. All results confirmed in 3 independent experiments. Data are shown as mean \pm SD. Two-tailed Student's $t$ test (A and F); 2 -way ANOVA with Dunnett's test (D, E, I, J, K, $\mathbf{M}$, and $\mathbf{P}-\mathbf{U})$. ${ }^{*} P<0.05,{ }^{* *} P<0.01$. 
we also examined CAMP expression in the NCBI's Gene Expression Omnibus (GEO) database (GSE13355, GSE30999, and GSE124161), and observed that $C A M P$ was significantly upregulated in human lesional psoriasis and wound skin biopsies (Figure 7, R and T, and Supplemental Figure 7L). We did not find any correlation between CAMP expression and alopecia areata, but BMP2 expression was strongly correlated with the degree of alopecia areata in human scalp biopsies (Figure 7U). Because of the important role of BMP signals in hair growth (30), the reduced BMP2 expression indicated aberrant BMP signals in human alopecia areata. In summary, these observations, together with the close physical location of dWAT to dermal components, suggest that mature dermal adipocytes are involved in maintaining skin morphology and function under both physiological and pathological conditions. Future mouse models that can specifically target dermal adipocytes, as well as more in-depth human studies, are certainly needed to better define the roles of dWAT.

\section{Discussion}

dWAT has received only a limited amount of attention compared with the more conventional fat pads studied in the field, such as the subcutaneous and epididymal fat pads. Recent studies have indicated the role of the mature and immature dermal adipocytes in a variety of processes, such as hair cycling, wound healing, and host defense $(22-24,45)$. However, dWAT is still poorly understood. In this study, we developed a protocol to isolate dermal adipocytes, then systemically characterized dWAT (Figures 1 and 2) and defined dWAT as a unique white fat pad that can undergo reversible dedifferentiation (Figures 4 and 5). Under normal conditions, the dWAT layer is thin. However, the expansion capacity of dWAT is quite potent in healthy obese mice; the thickness of the dWAT layer can reach more than $1 \mathrm{~mm}$ in Ob/Ob-MitoNEET-Tg mice. This is a notable amount of fat tissue in mice (Figure 3A). Owing to the specific location of dWAT, topical application of small-molecular compounds, such as PPAR $\gamma$ agonists, the adipocyte triglyceride lipase inhibitor atglistatin, and other agents, can easily modulate dWAT with minimal impact on other fat pads (Figure 3C and Supplemental Figure 2N). These findings indicate that dWAT can be manipulated selectively owing to its dynamic characteristics and easy accessibility. Furthermore, due to the anatomical location, one might suspect that dWAT may play a vital role in thermal insulation and response to cold exposure. However, we observed that dWAT is strongly resistant to "beiging," i.e., the induction of a thermally active phenotype (Figure 3E and Supplemental Figure $2 \mathrm{E})$. This suggests that dWAT has primarily other functions than thermogenesis. But given its anatomical location, dWAT may still contribute to thermoregulation through insulation.

In addition, dWAT and other components in the skin have strong mutual interactions, especially with respect to hair follicles $(4,8,9,24)$. As an active endocrine organ, the reciprocal signaling reactions between dWAT and hair follicles have drawn wide attention $(4,9,23,24)$. In addition to the signaling crosstalk, defects in fatty acid transporters can lead to skin barrier defects in both mice and humans, highlighting that fatty acids are essential for skin function (46). During the lipolytic action of the dedifferentiation process, it seems likely that dWAT provides a local source of fatty acids to other skin components. However, many reports demon- strated that mature adipocytes are not necessary for hair cycling per se $(4,15,23,24)$. Since we lack specific markers for dWAT to date, there are no genetic tools available to selectively manipulate dWAT. In addition, the mouse models used in these studies are germline mutants, or the genetic manipulations used do not specifically target adipocytes. Using the mouse models introduced here, we observed that mature dermal adipocytes inhibited the reinitiation of the anagen phase in depilated adult mice (Figure 7, A-J, and Supplemental Figure 7, A and C). This is consistent with a previous study showing that BMP signaling produced specifically by dermal adipocytes during early catagen inhibited stem cell activation during hair regeneration (30). However, we did not observe any changes in juvenile mice. Since the first 2 cycles of hair growth are highly synchronized in mice, the timeline in this early phase may have been imprinted during development and is lost at later stages. Beyond hair cycling, we found that juvenile mice displayed different levels of alopecia around weaning age upon HFD exposure, and suffered from broken hair shafts in the hair follicles (Supplemental Figure 7, F and G). Notably, the dermis became thicker as a consequence of the lack of dWAT (Figure 3I, Figure 7C, and Supplemental Figure 7A). This indicates a spatial balance between dWAT and dermis. Moreover, ablation of dermal adipocytes impaired wound healing (Figure 7K and Supplemental Figure 7, $\mathrm{H}$ and I). These observations suggest that mature dermal adipocytes are involved in maintaining hair integrity and the overall condition of the dermis, as well as skin integrity.

Despite the close adjacent location, we observed that mature dermal adipocytes expressed high levels of immune/inflammation response-related genes compared with subcutaneous adipocytes (Supplemental Figure 1, B and C). On the other hand, dermal adipocytes have much lower expression levels of genes related to cell adhesion and migration (Supplemental Figure 1D). So the 2 juxtaposed fat pads, dWAT and subcutaneous white adipose tissue (sWAT), show drastically distinct gene expression profiles. Except for the overall apparent gene expression differences with other fat pads, dermal adipocytes also showed distinct transcriptome profiles at different stages of hair cycling (Figure 2, B and C). It is a rather remarkable phenomenon that we can observe such dramatic transcriptional changes among adipocytes from the same anatomical location as a function of the hair cycle, while at the same time, gene expression in the neighboring subcutaneous fat pad is unlikely to undergo major transcriptional changes. These dWAT-specific changes must be a reflection of the intense paracrine crosstalk of the hair follicles and other local cell types with the adipocytes.

All of these findings reflect the multifunctional roles of mature dermal adipocytes, many of which we highlight here for the first time. Our observations offer a blueprint as to what aspects of the dermal adipocyte need to be further elucidated mechanistically to find out how mature adipocytes affect skin homeostasis and function. Importantly, for the first time to our knowledge, we have managed to perform gene expression analysis on highly purified dermal adipocytes. In the process of doing that, we identified Camp as a marker gene for dermal adipocytes, distinguishing dWAT from other fat depots (Figure 1, H and I). This provides the possibility of the generation of mouse models that can specifically target dWAT. Interestingly, 3T3-L1 cells, a widely used fibroblast cell line with strong adipogenic potential, also showed a high level of Camp 
expression upon differentiation. This suggests that 3T3-L1 cells may have originated as dermal adipocyte precursor cells (12).

According to previous studies, properly cultured mature adipocytes have the capacity to dedifferentiate into cells with multilineage potential $(19,20)$. In vivo, the dedifferentiation of terminally differentiated adipocytes was so far only observed in the mammary gland during lactation (21). In this study, we found that the mature dermal adipocytes also underwent strong dedifferentiation and redifferentiation during hair cycling (Figures 4 and 5). This is the second fat depot that we found to have a dynamic nature as well as the potential for dedifferentiation. Although no obvious differences were found in the transcriptome profile of dedifferentiated adipocytes versus adipocyte precursors that never differentiated previously, the cell cluster distribution and cell cycle analysis highlighted some critical differences. The dedifferentiated adipocytes were more sensitive to a redifferentiation protocol in vitro compared with adipogenic precursor cells that had not undergone a previous round of differentiation (Supplemental Figure 5, B and C). This very likely reflects epigenetic changes imprinted on these cells. There seems to be strong pressure on dermal adipocytes to commit to dedifferentiation rather than just enhanced lipolysis that would lead to a fully mature adipocyte devoid of a lipid droplet or an adipocyte committed to apoptosis. Notably, recent studies showed a link between dWAT loss and pathogenic dermal fibrosis $(23,37)$. As previously implicated (37), we formally show here that adiponectin-positive cells dedifferentiate and not only have the potential to redifferentiate, but can also transdifferentiate into myofibroblasts (Figure 6B). The appearance of myofibroblasts is now a well-established feature of fibrotic lesions and hypertrophic and keloid scars $(4,23,47)$. Our results indicate that the dermal adipocyte-myofibroblast transition is likely to be involved in the pathogenesis of fibrosis and scarring of the skin. Thus, interventions blocking the dedifferentiation of dermal adipocytes may represent a novel antiscarring and antifibrosis strategy. Since dWAT is an important component of the skin, it also represents a potential source of lipids, cytokines, and extracellular matrix $(4,23)$, which would have an impact on skin aging and quality (48). In view of the highly dynamic nature and significance of dWAT, as well as its reversible dedifferentiation capacity, there is ample opportunity in the future to selectively intervene in this layer of adipocytes to maintain skin homeostasis under physiological and pathophysiological conditions.

However, we need to take note that dWAT is different in mice and humans. Structurally, there is no panniculus carnosus layer that separates dWAT and subcutaneous fat in humans, and humans do not have hair cycling during season changes. Nevertheless, dWAT also shows a high degree of similarity between mice and humans in other areas. Studies had revealed 2 distinct adipose layers of human skin-associated adipose tissue. In humans, "fat islands" are evident in the dermis. They always appear around the pilosebaceous units, forming a cone geometry with a portion located in the dermis and another traversing the dermis to infiltrate the subcutaneous fat. Hair follicles root in these cone-structured adipocytes, and these "fat islands" are considered to be dWAT $(4,49)$. In humans, as the anagen phase is long, and hair follicles are highly asynchronous (mosaic cycling pattern), the oscillations of dWAT may not be easily detected. Nonetheless, there are reports showing that regions of hair loss are correlated with decreased adipocyte layer thickness in patients with alopecia areata or sebaceous nevus (27). The loss of dWAT was also observed in patients with systemic scleroderma $(26,28)$. These studies indicate that human dWAT may also be involved in hair cycling and skin fibrosis.

In addition, both mouse and human dWAT is the most superficial fat layer of the body; it may act as a thermal insulator to protect the heat generated from the body (49). Furthermore, studies in our inducible lipoatrophy mouse model and other mouse models indicated that dWAT is involved in wound healing in mice (22, 45). In humans, $A D I P O Q$ expression was significantly upregulated in normal wound skin, but significantly downregulated in keloid and lesional psoriasis skin as compared with normal control skin (Figure 7, Q and S, and Supplemental Figure 7, J and K). Fat grafting for the treatment of keloids and hypertrophic scars was associated with a better quality of scars (50). This suggests the important role of dWAT in human wound healing. As there are no mouse models that can specifically target dermal adipocytes, and there are no structures separating dWAT and subcutaneous fat in humans, it is hard to distinguish the roles of dWAT versus subcutaneous fat. But dWAT is adjacent to the dermis. Dermal adipocytes not only secrete a large amount of adipokines and extracellular matrix, but also provide a lot of fatty acids to the nearby components during regression. Therefore, they can well communicate with dermal components. Interestingly, we observed that dermal adipocytes were enriched for genes related to inflammation/immune/ defense responses and chemotaxis compared with subcutaneous adipocytes in mice. Among these genes, we found that Camp was exclusively enriched in dermal adipocytes compared with other kinds of adipocytes (Figure 1, H and I). As infection of skin stimulates the expansion of dWAT, and Camp has been reported to protect against invasive Staphylococcus aureus skin infection in mice (12), these data suggest a direct role of dWAT in maintaining skin barrier function. Cathelicidins are a family of antimicrobial peptides; they serve a critical role in mammalian innate immune defense against invasive bacterial infection. Lack of these antimicrobial peptides results in susceptibility to bacterial and viral infections (51). Human CAMP is an important antimicrobial peptide found in skin. It was reported to play a role in inflammation, infection, wound healing, mast cell chemotaxis, and angiogenesis $(42-44,51)$. Consistent with this, CAMP was significantly upregulated in human lesional psoriasis and wound skin as compared with normal skin (Figure 7, R and T, and Supplemental Figure 7L). Thus, dWAT may also serve as a unique component of the skin protecting from environmental challenges in humans.

In summary, our study highlights the importance of dWAT in both mouse and human settings, drawing more attention to this vastly ignored fat pad, and paving the path for additional dWAT research.

\section{Methods}

Animal models and treatment Mice were maintained on a 12-hour dark/12-hour light cycle and housed in groups of 3-5 with unlimited access to water and food. Normal chow diet was LabDiet 5058; doxycycline (Dox) chow diet $(200 \mathrm{mg} / \mathrm{kg}$ ) was D09050202i from Research Diets; high-fat diet (HFD; 60\%) was S1850, Paste, from Bio-Serv. The Institutional Animal Care and Use Committee of the University of Texas Southwestern Medical Center approved all animal experiments. All efforts were made to follow the Replacement, Refinement and Reduc- 
tion guidelines. To minimize discomfort, mice were anesthetized with a ketamine/xylazine cocktail or $2 \%-3 \%$ isoflurane during surgery or before organ harvest. All mice were on a pure C57BL/6J background. The Adiponectin-rtTA mouse was generated as previously described (52). TRE-Cre (JAX006234) and Rosa26-loxP-STOP-loxP-mT/mG (JAX007676) mouse lines were obtained from The Jackson Laboratory $(53,54)$. For lineage tracing studies, Adiponectin-rtTA mice were intercrossed to the TRE-Cre and Rosa26-loxP-STOP-loxP- $m T / m G$ mice; we refer to these triple-transgenic mice as AdipoChaser-mT/mG mice. The sex of the mice is specified in the figure legends.

\section{Tracing experiments}

Tracing de novo adipogenesis after the first anagen. We labeled the preexisting adipocytes generated from the first anagen. Pregnant mice were put on Dox (E18.5-P17), and skin was harvested at P17 from the offspring (pulse). Then mice were switched to normal chow diet, and skin was harvested at P30 (P30) or the normal chow diet was continued. Next, mice were fed with an HFD for 2 weeks (P63-P77) to induce the expansion of the dWAT layer, and we harvested skin at P77 (HFD). Alternatively, hair was depilated at P63 to induce reentry into anagen to induce the expansion of dWAT, and skin was harvested at P74-P77 (Depilation).

Tracing newly produced adipocytes after the second telogen induced by HFD or depilation. Mice were put on Dox chow diet for 7 days (P56P63), and skin was harvested at P63 (pulse). Then mice were switched to normal chow diet, and skin was harvested at P84 (Sham). Mice were fed with an HFD for 2 weeks (P70-P84) to induce the expansion of the dWAT layer, and skin was harvested at P84 (HFD); or hairs were depilated at P70 to induce reentry into anagen to induce the expansion of dWAT, and skin was harvested at P81-P84 (Depilation).

Tracing the fate of preexisting adipocytes at the second anagen. After 7 days of Dox chow diet (P28-P35), skins were harvested at P35 (Pulse). Thereafter, mice were fed with chow diet, and skin was harvested at P77 (Sham). Mice were fed with HFD for 2 weeks (P63-P77) to induce the expansion of the dWAT layer, and skin was harvested at P77 (HFD). Hairs were depilated at P63 to induce reentry into anagen to induce the expansion of dWAT, and skin was harvested at P74P77 (Depilation), or after the first depilation at P63. Hairs grew out; then the hair was again depilated at P100 and skin harvested around P112 ("twice depilation").

Tracing the natural turnover of dermal adipocytes. Mice were put on Dox chow diet for 7 days (P28-P35), and skins were harvested at P35 (pulse). Then mice were switched to normal chow diet, and skin was harvested at 7 months of age.

Other mouse models and treatment. Inducible fat-ablation (FAT-ATTAC) mice were generated and characterized by our laboratory (32). Two-month-old WT and their littermate FAT-ATTAC male mice were injected i.p. with dimerizer AP21087 (Invitrogen; $0.2 \mu \mathrm{g} / \mathrm{g} \mathrm{BW}$ ) or vehicle (0.4\% ethanol, $10 \%$ PEG-400, 2\% Tween-20 in water) every third day for 4 injections total. After the fourth injection, mice were shaved and chemically depilated for either hair cycling or wound healing experiments. The injection continued until the last time point of the experiment. For the hair cycling experiment, the mice were monitored daily 1 week after depilation. Tissues were harvested 11-14 days after depilation. For the wound healing experiment, the dorsal skin of each mouse, at the midline between the shoulder, was excised with a 6-mm biopsy punch (Miltex) to the level of the panniculus carnosus. A sterile circular silicone splint was attached to the skin with Krazy Glue, then secured with 5-0 PGA sutures. A topical antibiotic (bacitracin, polymyxin B, and neomycin) ointment was applied, and wounds were dressed with Tegaderm transparent dressing (3M) daily for the first 3 days. The wound size was measured at the indicated time points (55). For ablation of adipocytes in younger mice, WT and their littermate male FAT-ATTAC mice were injected i.p. with dimerizer (Invitrogen; $0.2 \mu \mathrm{g} / \mathrm{g} \mathrm{BW}$ ) or vehicle every third day from the age of 7 days until the age of 33 days. Hair cycling was monitored daily. Tissues were harvested at the indicated time points. Eight-week-old C57BL/6J male mice were put on HFD or chow diet for the indicated times. Skins were collected at the indicated time points to monitor the expansion of dWAT. Eight-week-old C57BL/6J male littermate mice were put on HFD or chow diet for 2 months, then mice were shaved and chemically depilated for hair cycling. C57BL/6J dams with 7-day-old offspring were put on HFD or chow diet. Each dam fed 4 male offspring with similar initial body weight. The hair cycling was observed daily. Tissues were harvested at indicated time points.

$\mathrm{C} 57 \mathrm{BL} / 6 \mathrm{~J}$ male mice were put into a $6^{\circ} \mathrm{C}$ cold chamber from 6 weeks old at indicated time points, and kept in cages individually and maintained on chow diet. Tissues were harvested for histology. Fiveto six-week-old C57BL/6J male mice were treated with $\beta_{3}$-agonist (CL316,243 , Sigma-Aldrich) at $1 \mathrm{mg} / \mathrm{kg} \mathrm{BW}$ daily by i.p. injections for 2 weeks while on a chow diet. Skin was harvested for histology, and subcutaneous fat was also harvested as positive controls. For observations related to dWAT during the first 2 cycles of hair growth, skin was harvested from 1-, 7-, 14-, 21-, 30-, and 50-day-old male mice separately. Two-month-old C57BL/6J male mice were kept in cages individually. Mice were shaved, and topically applied with vehicle $(2.5 \mu \mathrm{L}$ DMSO in $50 \mathrm{mg}$ CeraVe cream) or rosiglitazone (Sigma-Aldrich; $2.5 \mu \mathrm{L}$ rosiglitazone $2 \mu \mathrm{g} / \mu \mathrm{L}$ in $50 \mathrm{mg}$ CeraVe cream; total rosiglitazone applied: 0.1 $\mathrm{mg}$ ) daily for a total of 10 days. Skin was harvested for histology.

Two-month-old $\mathrm{Ob} / \mathrm{Ob}$ and $\mathrm{Ob} / \mathrm{Ob}$-MitoNEET-Tg male mice were on HFD for 10 weeks. $\mathrm{Ob} / \mathrm{Ob}$ mice were obtained from The Jackson Laboratory (JAX000632); the MitoNEET-Tg mice were generated as described previously (31). Skin was harvested for histology. Twentyeight-day-old C57BL/6J male mice were fed either normal drinking water or water containing $0.5 \%$ niacin (Sigma-Aldrich) for 1 and 3 weeks. The niacin water was exchanged daily. Skin was harvested for histology. Twenty-eight-day-old C57BL/6J male mice were topically applied with vehicle (Sigma-Aldrich; $5 \mu \mathrm{L}$ DMSO in $45 \mu \mathrm{L}$ acetone) or atglistatin (MilliporeSigma; $5 \mu \mathrm{L} 60 \mathrm{mM}$ atglistatin in $45 \mu \mathrm{L}$ acetone) twice daily for a total of 5 days. Skin was harvested for histology. Pparg $g^{f / f l}$ mice were bred with Adiponectin-Cre mice to generate the constitutive lipoatrophy

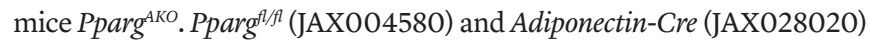
mice were obtained from The Jackson Laboratory $(56,57)$. Pparg ${ }^{f / f l}$ and Pparg ${ }^{A K O}$ male littermate mice were collected at P6.5, P14, and P21 for skin histology analysis. For skin fibrosis induced by bleomycin (SigmaAldrich), AdipoChaser-mT/mG male mice were put on Dox diet (P28P35) to label the dermal adipocytes of the second anagen. Then mice were switched to chow diet. At 2 months old, those mice were injected with $100 \mu \mathrm{L}$ vehicle ( $0.89 \%$ saline) or $100 \mu \mathrm{L}$ bleomycin $(1 \mathrm{mg} / \mathrm{mL})$ s.c. daily for a total of 3 weeks. Skin was subsequently harvested for histology. AdipoChaser-mT/mG male mice were put on Dox diet (P28-P35) to label the dermal adipocytes of the second anagen. Then mice were switched to chow diet. At 2 months of age, those mice were fed with either chow or HFD, and all the mice were injected with $50 \mathrm{mg} / \mathrm{kg} \mathrm{BrdU}$ (BD Biosciences) daily. Skin was harvested at day 7 for histology. 


\section{Single-cell RNA-Seq}

AdipoChaser-mTmG mice were fed with Dox chow diet (P28-P35), then switched to normal chow diet; skins were harvested around P56-P63. Single-cell suspensions were obtained from skin as described above. FACS-sorted CD31-CD45-PDGFR $\alpha^{+} \mathrm{GFP}^{-}$and CD31$\mathrm{CD} 45^{-} \mathrm{PDGFR} \alpha^{+} \mathrm{GFP}^{+}$cells were pooled together at a ratio of 1:1. A total of 15,000 cells were partitioned into droplets containing a barcoded bead, a single cell, and reverse transcription enzyme mix using the GemCode instrument (10x Genomics). Sample preparation followed the standard protocol as described previously (58). Libraries were sequenced on an Illumina NextSeq 500 High Output (400M) by the University of Texas Southwestern McDermott Center Next Generation Sequencing Core. Seventy-five paired-end reads were obtained using 1 flow cell with the following length input: $26 \mathrm{bp}$ read $1,66 \mathrm{bp}$ read 2, 0 bp index 1 , and 0 bp index 2 . Data were analyzed as described previously (58). Seurat (version 3) was used for cell cycle analysis (59).

\section{Additional methods}

Additional details on materials and methods are provided in Supplemental Methods online.

\section{Statistics}

All data were expressed as the mean \pm SD. We used GraphPad Prism 7.0 (GraphPad Software Inc.) or Microsoft Excel to perform the statistical analyses. For comparisons between 2 independent groups, a 2-tailed Student's $t$ test was used. For multiple comparisons, 1-way or 2-way ANOVA was used. $P$ less than 0.05 was considered statistically significant.

\section{Data and materials availability}

All data and materials supporting the findings of this study are available from the corresponding author upon request. All original microarray data were deposited in the NCBI's Gene Expression Omnibus database (GEO GSE128719, GSE134643, and GSE129371).

\section{Study approval}

The Institutional Animal Care and Use Committee of the University of Texas Southwestern Medical Center approved all animal experiments (APN:2015-101207G).

\section{Author contributions}

PES conceived the study. PES, Z Zhang, MS, and RKG designed the experiments. $Z$ Zhang and MS performed most of the experiments. SZ, YAA, YZ, MW, NL, Z Zi, and LG conducted some of the experiments. QZ, TO, NJ, and CMK helped with the breeding of mouse models. RG performed the metabolite measurement experiment. $\mathrm{CH}$ helped with the single-cell experiment. Z Zi performed the RNA-Seq data analysis. AK, CX, and $\mathrm{CH}$ performed the single-cell RNA-Seq analysis. ALG, CC, QAW, YD, LV, IK, and CMK gave useful suggestions to this study. Z Zhang, CMK, and PES wrote the manuscript, to which all authors contributed. Z Zhang was involved with designing the project, performed a majority of the experiments, and contributed to preparing the manuscript and is therefore listed before MS.

\section{Acknowledgments}

We thank all the members of the Scherer and Gupta laboratories for their support of this study. We also thank the University of Texas Southwestern Medical Center Metabolic Core Facility, McDermott Center Next Generation Sequencing Core and Bioinformatics Lab, Flow Cytometry Core of the Children's Research Institute, Histopathology Core, Live Cell Imaging Core, and Animal Resource Center; as well as Charlotte Lee for help with histology. This study was supported by American Heart Association Postdoctoral Award 16POST26420136 and Career Development Award 19CDA34670007 from the American Heart Association and the Harry S. Moss Heart Trust to MS; and NIH grants R01-DK104789, R56-DK119163, and R01DK119163 to RKG; RC2-DK118620 to PES and RKG; and R01DK55758, R01-DK099110, P01-DK088761, and P01-AG051459 to PES. PES was also supported by an unrestricted research grant from the Novo Nordisk Foundation.

Address correspondence to: Philipp E. Scherer, Touchstone Diabetes Center, Department of Internal Medicine, University of Texas Southwestern Medical Center, 5323 Harry Hines Boulevard, Dallas, Texas 75390-8549, USA. Phone: 214.648.8715; Email: Philipp. Scherer@utsouthwestern.edu.
1. Kershaw EE, Flier JS. Adipose tissue as an endocrine organ. JClin Endocrinol Metab. 2004;89(6):2548-2556.

2. Arner P, et al. Dynamics of human adipose lipid turnover in health and metabolic disease. Nature. 2011;478(7367):110-113.

3. Strawford A, Antelo F, Christiansen M, Hellerstein MK. Adipose tissue triglyceride turnover, de novo lipogenesis, and cell proliferation in humans measured with 2H2O. Am J Physiol Endocrinol Metab. 2004;286(4):E577-E588.

4. Kruglikov IL, Scherer PE. Dermal adipocytes: from irrelevance to metabolic targets? Trends Endocrinol Metab. 2016;27(1):1-10.

5. Lundbom J, Hakkarainen A, Lundbom N, Taskinen MR. Deep subcutaneous adipose tissue is more saturated than superficial subcutaneous adipose tissue. Int JObes (Lond). 2013;37(4):620-622.

6. Walker GE, et al. Deep subcutaneous adipose tissue: a distinct abdominal adipose depot. Obesity
(Silver Spring). 2007;15(8):1933-1943.

7. Cancello R, et al. Molecular and morphologic characterization of superficial- and deep-subcutaneous adipose tissue subdivisions in human obesity. Obesity (Silver Spring). 2013;21(12):2562-2570.

8. Driskell RR, Jahoda CA, Chuong CM, Watt FM, Horsley V. Defining dermal adipose tissue. Exp Dermatol. 2014;23(9):629-631.

9. Kruglikov IL, Scherer PE. Dermal adipocytes and hair cycling: is spatial heterogeneity a characteristic feature of the dermal adipose tissue depot? Exp Dermatol. 2016;25(4):258-262.

10. Wojciechowicz K, Gledhill K, Ambler CA, Manning $\mathrm{CB}$, Jahoda CA. Development of the mouse dermal adipose layer occurs independently of subcutaneous adipose tissue and is marked by restricted early expression of FABP4. PLoS One. 2013;8(3):e59811.

11. Kasza I, Hernando D, Roldán-Alzate A, Alexander CM, Reeder SB. Thermogenic profiling using magnetic resonance imaging of dermal and other adipose tissues. JCI Insight. 2016;1(13):e87146.

12. Zhang LJ, et al. Innate immunity. Dermal adipocytes protect against invasive Staphylococcus aureus skin infection. Science. 2015;347(6217):67-71.

13. Foster AR, Nicu C, Schneider MR, Hinde E, Paus R. Dermal white adipose tissue undergoes major morphological changes during the spontaneous and induced murine hair follicle cycling: a reappraisal. Arch Dermatol Res. 2018;310(5):453-462.

14. Rivera-Gonzalez GC, et al. Skin adipocyte stem cell self-renewal is regulated by a PDGFA/AKT-signaling axis. Cell Stem Cell. 2016;19(6):738-751.

15. Festa E, et al. Adipocyte lineage cells contribute to the skin stem cell niche to drive hair cycling. Cell. 2011;146(5):761-771.

16. Zhang B, et al. Hair follicles' transit-amplifying cells govern concurrent dermal adipocyte pro- 
duction through Sonic Hedgehog. Genes Dev. 2016;30(20):2325-2338

17. Nicu C, Hardman JA, Pople J, Paus R. Do human dermal adipocytes switch from lipogenesis in anagen to lipophagy and lipolysis during catagen in the human hair cycle? Exp Dermatol. 2019;28(4):432-435.

18. Singh $\mathrm{R}$, et al. Autophagy regulates adipose mass and differentiation in mice. JClin Invest. 2009;119(11):3329-3339.

19. Matsumoto T, et al. Mature adipocyte-derived dedifferentiated fat cells exhibit multilineage potential. J Cell Physiol. 2008;215(1):210-222.

20. Shen JF, Sugawara A, Yamashita J, Ogura H, Sato S. Dedifferentiated fat cells: an alternative source of adult multipotent cells from the adipose tissues. Int JOral Sci. 2011;3(3):117-124.

21. Wang QA, et al. Reversible de-differentiation of mature white adipocytes into preadipocytelike precursors during lactation. Cell Metab. 2018;28(2):282-288.e3.

22. Plikus MV, et al. Regeneration of fat cells from myofibroblasts during wound healing. Science. 2017;355(6326):748-752.

23. Rivera-Gonzalez G, Shook B, Horsley V. Adipocytes in skin health and disease. Cold Spring Harb Perspect Med. 2014;4(3):a015271.

24. Kruglikov IL, Zhang Z, Scherer PE. The role of immature mature adipocytes in hair cycling. Trends Endocrinol Metab. 2018;30(2):93-105.

25. Guerrero-Juarez CF, Plikus MV. Emerging nonmetabolic functions of skin fat. Nat Rev Endocrinol. 2018;14(3):163-173.

26. Varga J, Marangoni RG. Systemic sclerosis in 2016: dermal white adipose tissue implicated in SSc pathogenesis. Nat Rev Rheumatol. 2017;13(2):71-72.

27. Donati $G$, et al. Epidermal Wnt/ $\beta$-catenin signaling regulates adipocyte differentiation via secretion of adipogenic factors. Proc Natl Acad Sci US A. 2014;111(15):E1501-E1509.

28. Fleischmajer R, Damiano V, Nedwich A. Scleroderma and the subcutaneous tissue. Science. 1971;171(3975):1019-1021.

29. Ross SE, et al. Inhibition of adipogenesis by Wnt signaling. Science. 2000;289(5481):950-953.

30. Plikus MV, et al. Cyclic dermal BMP signalling regulates stem cell activation during hair regeneration. Nature. 2008;451(7176):340-344.

31. Kusminski CM, et al. MitoNEET-driven alterations in adipocyte mitochondrial activ- ity reveal a crucial adaptive process that preserves insulin sensitivity in obesity. Nat Med. 2012;18(10):1539-1549.

32. Pajvani UB, et al. Fat apoptosis through targeted activation of caspase 8: a new mouse model of inducible and reversible lipoatrophy. Nat Med. 2005;11(7):797-803.

33. Wang QA, Tao C, Gupta RK, Scherer PE. Tracking adipogenesis during white adipose tissue development, expansion and regeneration. Nat Med. 2013;19(10):1338-1344.

34. Berry R, Jeffery E, Rodeheffer MS. Weighing in on adipocyte precursors. Cell Metab. 2014;19(1):8-20.

35. Yamamoto T, et al. Animal model of sclerotic skin. I: Local injections of bleomycin induce sclerotic skin mimicking scleroderma. J Invest Dermatol.1999;112(4):456-462.

36. Varga J, Abraham D. Systemic sclerosis: a prototypic multisystem fibrotic disorder. J Clin Invest. 2007;117(3):557-567.

37. Marangoni RG, et al. Myofibroblasts in murine cutaneous fibrosis originate from adiponectin-positive intradermal progenitors. Arthritis Rheumatol. 2015;67(4):1062-1073.

38. Santiago B, et al. Topical application of a peptide inhibitor of transforming growth factor- $\beta 1$ ameliorates bleomycin-induced skin fibrosis. JInvest Dermatol. 2005;125(3):450-455.

39. Karnik P, et al. Hair follicle stem cell-specific PPAR $\gamma$ deletion causes scarring alopecia. J Invest Dermatol. 2009;129(5):1243-1257.

40. Sardella C, et al. Delayed hair follicle morphogenesis and hair follicle dystrophy in a lipoatrophy mouse model of Pparg total deletion. J Invest Dermatol. 2018;138(3):500-510.

41. Wang F, Mullican SE, DiSpirito JR, Peed LC, Lazar MA. Lipoatrophy and severe metabolic disturbance in mice with fat-specific deletion of PPAR $\gamma$. Proc Natl Acad Sci U S A. 2013;110(46):18656-18661.

42. Niyonsaba F, et al. A cathelicidin family of human antibacterial peptide LL-37 induces mast cell chemotaxis. Immunology. 2002;106(1):20-26.

43. Carretero $\mathrm{M}$, et al. In vitro and in vivo wound healing-promoting activities of human cathelicidin LL-37. J Invest Dermatol. 2008;128(1):223-236.

44. Koczulla R, et al. An angiogenic role for the human peptide antibiotic LL-37/hCAP-18. JClin Invest. 2003;111(11):1665-1672.

45. Schmidt BA, Horsley V. Intradermal adipocytes mediate fibroblast recruitment during skin wound healing. Development. 2013;140(7):1517-1527.

46. Khnykin D, Miner JH, Jahnsen F. Role of fatty acid transporters in epidermis: Implications for health and disease. Dermatoendocrinol. 2011;3(2):53-61.

47. Darby IA, Laverdet B, Bonté F, Desmoulière A. Fibroblasts and myofibroblasts in wound healing. Clin Cosmet Investig Dermatol. 2014;7:301-311.

48. Kruglikov IL, Scherer PE. Skin aging as a mechanical phenomenon: the main weak links. Nutr Healthy Aging. 2018;4(4):291-307.

49. Alexander CM, et al. Dermal white adipose tissue: a new component of the thermogenic response. J Lipid Res. 2015;56(11):2061-2069.

50. Lee G, Hunter-Smith DJ, Rozen WM. Autologous fat grafting in keloids and hypertrophic scars: a review. Scars Burn Heal. 2017;3:2059513117700157.

51. Ong PY, et al. Endogenous antimicrobial peptides and skin infections in atopic dermatitis. $N$ Engl J Med.2002;347(15):1151-1160.

52. Sun K, et al. Dichotomous effects of VEGF-A on adipose tissue dysfunction. Proc Natl Acad Sci US A. 2012;109(15):5874-5879.

53. Perl AK, Wert SE, Nagy A, Lobe CG, Whitsett JA Early restriction of peripheral and proximal cell lineages during formation of the lung. Proc Natl Acad Sci U S A. 2002;99(16):10482-10487.

54. Muzumdar MD, Tasic B, Miyamichi K, Li L, Luo L. A global double-fluorescent Cre reporter mouse. Genesis. 2007;45(9):593-605.

55. Wang X, Ge J, Tredget EE, Wu Y. The mouse excisional wound splinting model, including applications for stem cell transplantation. Nat Protoc. 2013;8(2):302-309.

56 . He W, et al. Adipose-specific peroxisome proliferator-activated receptor gamma knockout causes insulin resistance in fat and liver but not in muscle. Proc Natl Acad Sci U S A. 2003;100(26):15712-15717.

57. Eguchi J, et al. Transcriptional control of adipose lipid handling by IRF4. Cell Metab. 2011;13(3):249-259.

58. Hepler C, et al. Identification of functionally distinct fibro-inflammatory and adipogenic stromal subpopulations in visceral adipose tissue of adult mice. Elife. 2018;7:e39636.

59. Butler A, Hoffman P, Smibert P, Papalexi E, Satija R. Integrating single-cell transcriptomic data across different conditions, technologies, and species. Nat Biotechnol.2018;36(5):411-420. 